\title{
THE TIME-DEPENDENT FX-SABR MODEL: EFFICIENT CALIBRATION BASED ON EFFECTIVE PARAMETERS
}

\author{
ANTHONIE W. VAN DER STOEP*,†, ${ }^{*}$ LECH A. GRZELAK*,‡ \\ and CORNELIS W. OOSTERLEE ${ }^{\dagger, \ddagger}$ \\ *Pricing Model Validation, Rabobank \\ Graadt van Roggenweg 400, 3531 AH \\ Utrecht, The Netherlands \\ ${ }^{\dagger}$ CWI - National Research Institute for Mathematics and Computer Science \\ Science Park 123, 1098 XG, Amsterdam, The Netherlands \\ $\ddagger_{D}$ Delft Institute of Applied Mathematics \\ Delft University of Technology, Mekelweg 4, 2628 CD \\ Delft, The Netherlands \\ §A.W.van.der.Stoep@cwi.nl \\ Received 31 October 2014 \\ Accepted 26 May 2015 \\ Published 10 September 2015
}

\begin{abstract}
We present a framework for efficient calibration of the time-dependent SABR model (Fernández et al. (2013) Mathematics and Computers in Simulation 94, 55-75; Hagan et al. (2002) Wilmott Magazine 84-108; Osajima (2007) Available at SSRN 965265.) in an foreign exchange (FX) context. In a similar fashion as in (Piterbarg (2005) Risk 18 (5), 71-75) we derive effective parameters, which yield an accurate and efficient calibration. On top of the calibrated FX-SABR model, we add a non-parametric local volatility component, which naturally compensates for possible calibration errors. By means of Monte Carlo pricing experiments, we show that the time-dependent FX-SABR model enables an accurate and consistent pricing of barrier options and outperforms the constant-parameter SABR model and the traditional local volatility model (Derman \& Kani (1998) International Journal of Theoretical and Applied Finance 1 (1), 61110; Dupire (1994) Risk 7 (1), 18-20). We also discuss the role of the local volatility component in pricing barrier options.
\end{abstract}

Keywords: Time-dependent SABR; FX; calibration; effective parameters; local volatility; Monte Carlo; path-dependent.

$\S$ Corresponding author. 


\section{Introduction}

Over the last decades, the foreign exchange (FX) market has rapidly grown to become the world's largest and most liquid OTC market (Weithers 2011). ${ }^{\text {a }}$ According to the latest triennial survey by the Bank for International Settlements, ${ }^{\mathrm{b}}$ an estimated $\$ 5.3$ trillion changes hands every day. FX swaps are the most actively traded instruments, followed by spot trading. ${ }^{\mathrm{c}}$

The fast expansion of the FX market, together with the rise of complex products like the Power-Reverse Dual-Currency (Sippel \& Ohkoshi 2002) and the EquityCMS Chameleon, led to an increasing demand for modeling FX rates in a sophisticated way. As a consequence, the literature on modeling FX rates is plentiful and many stochastic models are available. In the financial industry, typically a three-factor pricing model is used (Frey \& Sommer 1996, Piterbarg 2006, Sippel \& Ohkoshi 2002), where FX dynamics are assumed to be lognormal and the domestic and foreign interest rates follow a Hull-White one factor Gaussian model (Hull \& White 1993). This model is not capable of generating a skew effect which we observe in the FX market. In order to overcome this issue, several researchers have applied local volatility (Piterbarg 2006), stochastic volatility and displaced diffusion models (Kawai \& Jäckel 2007) in an FX context. In Grzelak \& Oosterlee (2012) and Van Haastrecht \& Pelsser (2011), the FX dynamics are given by the Heston model and the Schöbel-Zhu model, respectively.

The pricing and hedging of complex path-dependent financial products requires an accurate calibration to prices of European-type options with different expiries, which contain information about market behavior through time. The model should also reflect realistic implied volatility smile dynamics, both with respect to the forward smile (Tataru \& Fisher 2010) and, second, the underlying.

The payoff of a path-dependent product is determined by the evolution of the underlying through time, i.e. its price depends on the transition densities from one future state to another (Baker et al. 2004). The future transition densities implied by a particular model are reflected by the forward implied volatility smiles it produces. Although the local volatility model (Derman \& Kani 1998, Dupire 1994) can be calibrated perfectly to any set of arbitrage-free European-type option prices, it exhibits a flattening of the forward smile. This may lead to a mispricing of products which are sensitive to the forward implied volatility skew, like forward starting options, cliquets and path-dependent products. Alternatives for pricing such contracts are stochastic volatility models, which predict that the forward smile has a comparable shape as the smile observed today and typically yield more accurate results (Baker et al. 2004, Engelmann et al. 2011, Gatheral 2011).

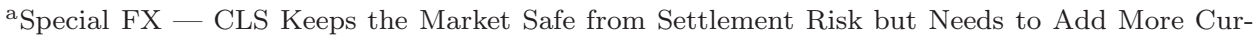
rencies. The Economist, September 2013.

${ }^{\mathrm{b}}$ Triennial Central Bank Survey - Foreign Exchange Turnover in April 2013: Preliminary Global Results. Technical report, Bank for International Settlements, September 2013.

${ }^{\mathrm{c}}$ Spot trading is characterized by immediate delivery of a foreign currency or commodity "on the spot", as opposed to futures contracts, which typically expire before a physical delivery.
} 
The "local dynamics" of the implied volatility smile, i.e. with respect to the underlying, are also relevant, especially for hedging purposes. One of the main motivations for Hagan et al. (2002) to introduce the SABR model is the typically inaccurate smile moves as the underlying changes predicted by the local volatility model. In particular, this model predicts that the smile shifts to higher prices as the underlying moves to lower prices, which is more extreme than market behavior or may be even opposite to it, resulting in unstable hedges (Baker et al. 2004, Hagan et al. 2002, Johnson \& Lee 2003). In contrast, the smile implied by the SABR model "follows" the underlying. ${ }^{\mathrm{d}}$

On the base of the previous discussion, we consider the time-dependent SABR model in an FX context. As this model involves time-dependent parameters, it allows for calibration to European-type option prices with different expiries. Furthermore, compared to the local volatility model, it yields more realistic forward implied volatility smiles and thirdly, this model is able of capturing the smile dynamics with respect to the underlying more accurately.

In Hagan et al. (2002), the time-dependent SABR model was already presented. Osajima (2007) derives an asymptotic expression for the implied volatility. Furthermore, he introduces a new "FX hybrid SABR model" and gives an asymptotic expansion formula for implied volatilities. Fernández et al. (2013) apply GPU technology for the Monte Carlo calibration of the static and time-dependent SABR models. They assume a time-dependent vol-vol parameter and correlation under the condition that these parameters decrease over time. In Glasserman \& Wu (2011), the authors assume piecewise-constant parameters and show how the asymptotic expansion of the bivariate transition density of the underlying and its stochastic volatility presented in $\mathrm{Wu}$ (2012) is used in the calibration. Further, in Larsson (2010), a closed-form approximation of the option price for the time-dependent $\mathrm{SABR}$ model is derived.

From a theoretical point of view, implied volatility expansion formulas as in e.g. (Larsson 2010, Osajima 2007) yield highly efficient calibration. However, in a practical sense, these formulas typically only work under certain parameter conditions.

In this paper, we calibrate the time-dependent SABR model by means of effective parameters. Effective parameters can be considered as "sophisticated averages" of the corresponding time-dependent parameters. In Piterbarg (2005) the effective parameters approach has been followed with respect to the time-dependent displaced diffusion stochastic volatility model. In a similar fashion, we derive effective parameters by considering the qualitative effects of the SABR parameters on the shape of the implied volatility smile. By means of numerical experiments, we show that our approach yields an accurate and efficient calibration. Moreover, the idea

\footnotetext{
${ }^{\mathrm{d}}$ Rebonato (2005) supports the conclusions of Hagan et al. regarding the dynamics of the smile with respect to the underlying in an FX context, but he points out that these may not be valid when stochastic interest rates are involved.
} 
behind effective parameters is intuitive and the resulting formulas are straightforward and relatively easy to implement.

As the calibration may not be perfect due to e.g. possible inaccuracies in mapping time-dependent to effective parameters, we add a non-parametric local volatility component (see e.g. Ren et al. 2007), which "bridges" the mismatch between the prices in the market and the ones implied by the calibrated model.

The present paper is organized as follows. In Sec. 2, we present the dynamics of the time-dependent FX-SABR model. We also specify the non-parametric local volatility component. In Sec. 3, we describe the calibration problem for the timedependent SABR model and in which way effective parameters facilitate efficient calibration. Thereafter, in Secs. 4.1-4.3, we state results regarding the effective vol-vol, term structure and correlation parameters, respectively. Subsequently, we calibrate the time-dependent SABR model in Sec. 5. We show that the local volatility component yields an enhancement in results. Further, we price standard barrier options and compare results for the constant-parameter FX-SABR model, the local volatility model and the time-dependent FX-SABR model. We also discuss the role of the local volatility component. Section 6 concludes.

\section{Time-Dependent FX-SABR Model with Local Volatility}

In this section, we present the time-dependent FX-SABR model. As the spot dynamics involve time-dependent zero-coupon bonds, we cannot directly apply Hagan's formulas. We resolve this issue by fixing the expiry of the zero-coupon bonds at the largest time to maturity, which represents the terminal payment date.

We first calibrate the time-dependent FX-SABR model. Subsequently we add a non-parametric local volatility component, which compensates for possible calibration inaccuracies. The local volatility component can compensate for any calibration error, which is a consequence of the mimicking theorem of (Gyöngy 1986). ${ }^{\mathrm{e}}$ A perfect fit to a European-type option price can be obtained, as its price is determined by the distribution of the underlying at a particular point in time.

Despite this feature of the local volatility component, the stochastic volatility parameters need to be calibrated accurately. European-type option prices with different expiries provide insight in market behavior over time and our target is to "capture" this information in the model by replicating these prices. Further, for the hedging and pricing of path-dependent products, a model should reflect realistic smile dynamics, both with respect to the forward implied volatility smile and regarding changes in the underlying. As the SABR model, compared to the local volatility model, typically captures these features more accurately (Baker et al. 2004, Gatheral 2011, Hagan et al. 2002, Johnson \& Lee 2003), we reduce the contribution of the local volatility component by an accurate calibration.

eThe theorem states that given a general Itô process, a Markov process containing a local volatility component with the same marginal distributions as the former exists. 


\subsection{Time-dependent $F X-S A B R$ model}

Let $r_{d}(t)$ and $r_{f}(t)$ denote deterministic domestic and foreign interest rates, respectively, and $M_{d}(t)$ and $M_{f}(t)$ are corresponding moneyness accounts, determined by

$$
\mathrm{d} M_{d}(t)=r_{d}(t) M_{d}(t) \mathrm{d} t, \quad \mathrm{~d} M_{f}(t)=r_{f}(t) M_{f}(t) \mathrm{d} t .
$$

Let $y(t)$ be the spot FX, expressed in units of domestic currency per unit of a foreign currency. Further, define

$$
\begin{aligned}
& P_{d}(t, T):=M_{d}(t) \mathbb{E}^{\mathbb{Q}}\left[\frac{1}{M_{d}(T)} \mid \mathcal{F}(t)\right], \\
& P_{f}(t, T):=M_{f}(t) \mathbb{E}^{\mathbb{Q}}\left[\frac{1}{M_{f}(T)} \mid \mathcal{F}(t)\right],
\end{aligned}
$$

as the domestic and foreign zero-coupon bonds, respectively, see e.g. (Musiela \& Rutkowski 2006, Piterbarg 2006).

The time-dependent $F X$-SABR model assumes the following dynamics under the domestic risk-neutral $\mathbb{Q}$-measure:

$$
\begin{aligned}
\mathrm{d} y(t)= & \left(r_{d}(t)-r_{f}(t)\right) y(t) \mathrm{d} t \\
& +\omega(t) \sigma(t)\left(\frac{P_{d}(t, T)}{P_{f}(t, T)}\right)^{1-\beta} y^{\beta}(t) \mathrm{d} W_{y}^{\mathbb{Q}}(t), \quad y(0)=y_{0}, \\
\mathrm{~d} \sigma(t)= & \gamma(t) \sigma(t) \mathrm{d} W_{\sigma}^{\mathbb{Q}}(t), \quad \sigma(0)=1,
\end{aligned}
$$

with $\mathrm{d} W_{y}^{\mathbb{Q}}(t) \mathrm{d} W_{\sigma}^{\mathbb{Q}}(t)=\rho_{y, \sigma}(t) \mathrm{d} t$ and $\rho_{y, \sigma}(t), \gamma(t)$ and $\beta$ denoting the correlation, vol-vol parameter and skew parameter, respectively. Further, $T$ denotes the time to maturity. The skew parameter is typically set at $\beta=0.5$ (Rebonato et al. 2011). For calibration purposes, the volatility dynamics are scaled, which introduces the term structure parameter $\omega(t)$.

As this paper aims to apply the SABR model in an FX context, it is convenient to price under the forward measure. The traditional SABR model describes the dynamics of the forward under the corresponding forward measure. Assuming a grid of $N$ expiries $T_{i}, 1,2, \ldots, N$, the FX forward $y^{T_{i}}(t):=y(t) \frac{P_{f}\left(t, T_{i}\right)}{P_{d}\left(t, T_{i}\right)}$ is a martingale under the domestic $T_{i}$-forward measure and the SABR model consistently prices an option with expiry $T_{i}$. However, simultaneous pricing of options with different expiries raises consistency issues when using a single set of time-dependent parameters. We resolve this by writing the dynamics of $y^{T_{i}}(\cdot)$ with respect to $T_{N}$, which represents the terminal payment date. ${ }^{\mathrm{f}}$

More concretely, let $T_{1}, T_{2}, \ldots, T_{N}$ be a set of expiries and suppose that the spot dynamics (2.1) and (2.2) involve the zero-coupon bonds $P_{d}\left(t, T_{N}\right)$ and $P_{f}\left(t, T_{N}\right)$.

${ }^{\mathrm{f}}$ A similar approach was followed in Glasserman \& Wu (2011). The authors in Chen et al. (2012) resolve the issue in a stochastic interest rates framework by projecting the volatility term on a lognormal distribution, which yields forward dynamics that are in the desired SABR form. 
The dynamics of the forward $y^{T_{i}}(t)$ corresponding to an arbitrary expiry $T_{i}$ then read:

$$
\begin{aligned}
\mathrm{d} y^{T_{i}}(t) & =\mathrm{d}\left(y(t) \frac{P_{f}\left(t, T_{i}\right)}{P_{d}\left(t, T_{i}\right)}\right) \\
& =\frac{P_{f}\left(t, T_{i}\right)}{P_{d}\left(t, T_{i}\right)} \mathrm{d} y(t)+\left(r_{f}(t)-r_{d}(t)\right) y(t) \frac{P_{f}\left(t, T_{i}\right)}{P_{d}\left(t, T_{i}\right)} \mathrm{d} t .
\end{aligned}
$$

Substituting the dynamics of $y(t)$ in $(1)$ yields $^{\mathrm{g}}$ :

$$
\mathrm{d} y^{T_{i}}(t)=\left(\frac{P_{f}\left(t, T_{i}\right)}{P_{d}\left(t, T_{i}\right)}\right)^{\beta}\left(\frac{P_{f}\left(t, T_{i}\right)}{P_{d}\left(t, T_{i}\right)}\right)^{1-\beta} \omega(t) \sigma(t)\left(\frac{P_{d}\left(t, T_{N}\right)}{P_{f}\left(t, T_{N}\right)}\right)^{1-\beta} y^{\beta}(t) \mathrm{d} W_{y}^{T_{i}}(t) .
$$

By definition of the zero-coupon bond, we have for deterministic interest rates $P\left(T_{i}, T_{N}\right)=\frac{P\left(t, T_{N}\right)}{P\left(t, T_{i}\right)}$, which gives:

$$
\mathrm{d} y^{T_{i}}(t)=\omega(t) \sigma(t)\left(\frac{P_{d}\left(T_{i}, T_{N}\right)}{P_{f}\left(T_{i}, T_{N}\right)}\right)^{1-\beta}\left(y^{T_{i}}(t)\right)^{\beta} \mathrm{d} W_{y}^{T_{i}}(t)
$$

Scaling the forward dynamics results in the following model:

$$
\begin{aligned}
\mathrm{d} y^{T_{i}}(t) & =\omega_{1}(t) \sigma(t)\left(y^{T_{i}}(t)\right)^{\beta} \mathrm{d} W_{y}^{T_{i}}(t), \quad y^{T_{i}}(0)=: \bar{y}_{0}^{T_{i}}=1, \\
\mathrm{~d} \sigma(t) & =\gamma(t) \sigma(t) \mathrm{d} W_{\sigma}^{T_{i}}, \quad \sigma(0)=1,
\end{aligned}
$$

with $\mathrm{d} W_{y}^{T_{i}}(t) \mathrm{d} W_{\sigma}^{T_{i}}(t)=\rho_{y, \sigma}(t) \mathrm{d} t$ and

$$
\omega_{1}(t):=\omega(t)\left(\frac{P_{d}\left(T_{i}, T_{N}\right)}{y_{0}^{T_{i}} P_{f}\left(T_{i}, T_{N}\right)}\right)^{1-\beta}
$$

As model (2.3)-(2.4) is in "SABR form", we can apply Hagan's formulas under the assumption of constant parameter values. This is particularly a useful property for the calibration framework we propose, which is based on effective parameters (see Sec. 3). The additional constants $P_{d}\left(T_{i}, T_{N}\right)$ and $P_{f}\left(T_{i}, T_{N}\right)$ in $(2.5)$ allow for the calibration of model $(2.1)$ and (2.2) across multiple expiries. Without these terms, the spot dynamics are forward dependent, i.e. from the forward dynamics of $y^{T_{i}}(\cdot)$ and $y^{T_{j}}(\cdot), i \neq j$ different dynamics for the spot FX and thus different models can be derived.

For the sake of notation, $y^{T_{i}}(t)$ and $\sigma(t)$ denote the scaled forward and volatility dynamics, respectively, unless otherwise mentioned. An exception holds for the initial forward: $y_{0}^{T_{i}}$ denotes the original initial forward and $\bar{y}_{0}^{T_{i}}=1$ corresponds to the model with scaled forward dynamics.

g As the Radon-Nikodym derivative (Geman et al. 1995) is $\Lambda_{\mathbb{Q}}^{T_{i}}=\frac{P_{d}\left(t, T_{i}\right) M(0)}{P_{d}\left(0, T_{i}\right) M(t)}=1$, we have $\mathrm{d} W_{y}^{T_{i}}(t)=\mathrm{d} W_{y}^{\mathbb{Q}}(t)$. 


\subsection{Local volatility compensator}

Calibration of model (2.1) and (2.2) may not be perfect. For this reason, we add a non-parametric local volatility component that acts as a "compensator" for the mismatch between the market and calibrated model prices.

Adding the local volatility component $\sigma_{\mathrm{SLV}}(t, y(t))$ to model (2.1) and (2.2) yields the following $y(t)$ dynamics:

$$
\begin{aligned}
\mathrm{d} y(t)= & \left(r_{d}(t)-r_{f}(t)\right) y(t) \mathrm{d} t \\
& +\sigma_{\mathrm{SLV}}(t, y(t)) \omega(t) \sqrt{V(t)}\left(\frac{P_{d}(t, T)}{P_{f}(t, T)}\right)^{1-\beta} y^{\beta}(t) \mathrm{d} W_{y}^{\mathbb{Q}}(t),
\end{aligned}
$$

where $V(t)$ denotes variance to avoid double use of the letter $\sigma$. Defining ${ }^{\text {h }}$

$$
\psi(y(t), V(t)):=\omega(t) \sqrt{V(t)}\left(\frac{P_{d}(t, T)}{P_{f}(t, T)}\right)^{1-\beta} y^{\beta-1}(t),
$$

the spot dynamics are given by:

$$
\begin{aligned}
\mathrm{d} y(t) / y(t) & =\left(r_{d}(t)-r_{f}(t)\right) \mathrm{d} t+\sigma_{\mathrm{SLV}}(t, y(t)) \psi(y(t), V(t)) \mathrm{d} W_{y}^{\mathbb{Q}}(t), \quad y(0)=y_{0}, \\
\mathrm{~d} V(t) & =\gamma^{2}(t) V(t) \mathrm{d} t+2 \gamma(t) V(t) \mathrm{d} W_{\sigma}^{\mathbb{Q}}(t), \quad V(0)=1,
\end{aligned}
$$

with $\mathrm{d} W_{y}^{\mathbb{Q}}(t) \mathrm{d} W_{\sigma}^{\mathbb{Q}}(t)=\rho_{y, \sigma}(t) \mathrm{d} t$.

In Van der Stoep et al. (2014) we applied the Tanaka-Meyer formula (Tanaka 1963) and well-known relations between the option price and the underlying's marginal density based on the mimicking theory of Gyöngy (Gatheral 2011, Gyöngy 1986) to obtain the following result:

$$
\sigma_{\mathrm{SLV}}^{2}(t, K)=\frac{\sigma_{\mathrm{LV}}^{2}(t, K)}{\mathbb{E}\left[\psi^{2}(y(t), V(t)) \mid y(t)=K\right]} .
$$

Here, $\sigma_{\mathrm{LV}}^{2}(\cdot)$ denotes Dupire's local volatility term (Dupire 1994), which is either expressed in terms of European call prices or implied volatilities $\bar{\sigma}(\cdot)$. In the numerical experiments, we choose the latter:

$$
\begin{aligned}
& \sigma_{\mathrm{LV}}^{2}(t, K)= \\
& \left.\quad \frac{\bar{\sigma}^{2}(T, K)+2 \bar{\sigma}(T, K) T\left(\frac{\partial \bar{\sigma}(T, K)}{\partial T}+r K \frac{\partial \bar{\sigma}(T, K)}{\partial K}\right)}{\left(1-\frac{K y}{\bar{\sigma}(T, K)} \frac{\partial \bar{\sigma}(T, K)}{\partial K}\right)^{2}+K \bar{\sigma}(T, K) T\left(\frac{\partial \bar{\sigma}(T, K)}{\partial K}-\frac{1}{4} K \bar{\sigma}(T, K) T\left(\frac{\partial \bar{\sigma}(T, K)}{\partial K}\right)^{2}+K \frac{\partial^{2} \bar{\sigma}(T, K)}{\partial K^{2}}\right)}\right|_{T=t} .
\end{aligned}
$$

\footnotetext{
${ }^{\mathrm{h}}$ For this model $\psi(\cdot)$ also depends on the underlying, $y(t)$, in contrast to the Heston SLV model discussed in Van der Stoep et al. (2014). As we condition on $y(t)=K$ though, this issue is resolved in a natural way.
} 
Substituting (6) yields

$$
\begin{aligned}
\sigma_{\mathrm{SLV}}^{2}(t, K) & =\frac{\sigma_{\mathrm{LV}}^{2}(t, K)}{\mathbb{E}\left[\left(\omega(t) \sqrt{V(t)}\left(\frac{P_{d}(t, T)}{P_{f}(t, T)}\right)^{1-\beta} y^{\beta-1}(t)\right)^{2} \mid y(t)=K\right]} \\
& =\frac{\sigma_{\mathrm{LV}}^{2}(t, K)}{\omega^{2}(t)\left(\frac{P_{d}(t, T)}{P_{f}(t, T)}\right)^{2-2 \beta} K^{2 \beta-2} \mathbb{E}[V(t) \mid y(t)=K]}
\end{aligned}
$$

The conditional expectation appearing in (2.10) can be evaluated efficiently by the non-parametric method presented in Van der Stoep et al. (2014).

\section{Calibration Problem}

In this section, we discuss an important target of this paper, namely the calibration of model (2.1) and (2.2). For this, we need to price basic options on the FX rate with discounted value

$$
C(t, T, K)=\mathbb{E}^{\mathbb{Q}}\left[\frac{M_{d}(t)}{M_{d}(T)}(y(T)-K)^{+} \mid \mathcal{F}(t)\right],
$$

where $\mathbb{Q}$ denotes the domestic risk-neutral measure. As we mentioned at the beginning of Sec. 2, we will calibrate model (2.1) and (2.2) as accurately as possible in order to enable an accurate and consistent pricing and hedging of path-dependent and other forward volatility sensitive products.

In the calibration, we make use of effective parameters, which are "sophisticated averages" of their corresponding time-dependent counterparts. In this section, we discuss how effective parameters facilitate efficient calibration.

The effective FX-SABR model is given by the following dynamics under the $T_{i}$-forward measure, $i=1, \ldots, N$ :

$$
\begin{aligned}
\mathrm{d} \widetilde{y}^{T_{i}}(t) & =\widetilde{\omega}_{1} \widetilde{\sigma}(t)\left(\widetilde{y}^{T_{i}}(t)\right)^{\beta} \mathrm{d} W_{y}^{T_{i}}(t), \quad \widetilde{y}^{T_{i}}(0)=\bar{y}_{0}^{T_{i}}=1, \\
\mathrm{~d} \widetilde{\sigma}(t) & =\widetilde{\gamma} \widetilde{\sigma}(t) \mathrm{d} W_{\sigma}^{T_{i}}(t), \quad \widetilde{\sigma}(0)=1,
\end{aligned}
$$

with $\mathrm{d} W_{y}^{T_{i}}(t) \mathrm{d} W_{\sigma}^{T_{i}}(t)=\widetilde{\rho}_{y, \sigma} \mathrm{d} t$ and

$$
\widetilde{\omega}_{1}:=\widetilde{\omega}\left(\frac{P_{d}\left(T_{i}, T_{N}\right)}{y_{0}^{T_{i}} P_{f}\left(T_{i}, T_{N}\right)}\right)^{1-\beta}, \quad \widetilde{\omega}:=\sigma_{0} .
$$

This is just the constant-parameter version of model (2.3) and (2.4) derived in Sec. 2 .

Remark 3.1. We have defined model (2.3) and (2.4) such that $\bar{y}_{0}^{T_{i}}=1$. Therefore, the formulas in this section can be simplified. However, for the sake of completeness we include the term $\bar{y}_{0}^{T_{i}}$. 


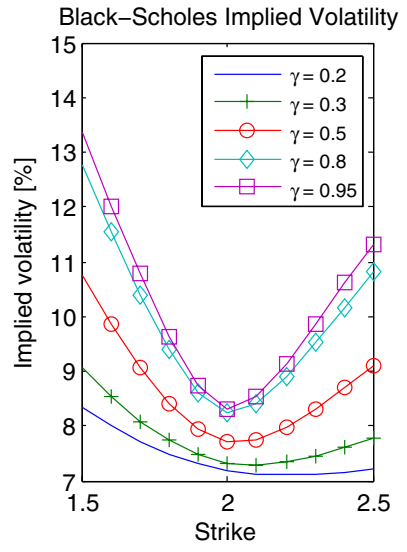

(a)

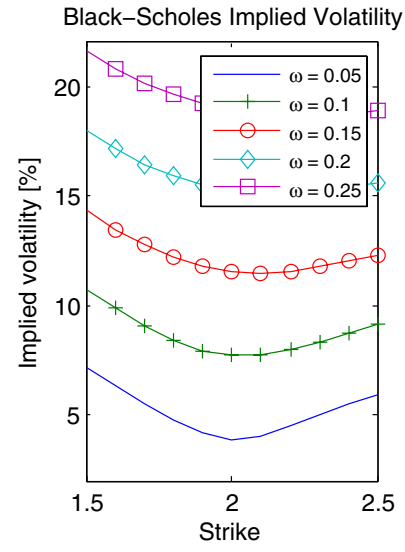

(b)

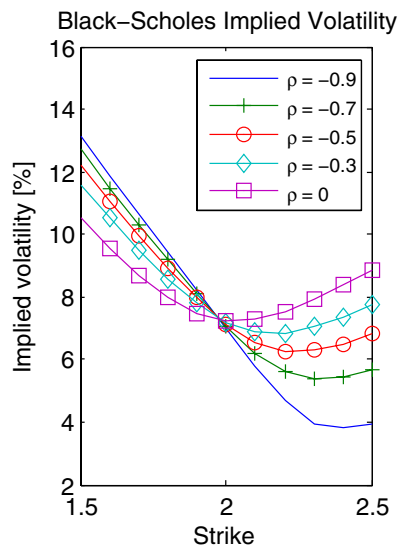

(c)

Fig. 1. Effect of vol-vol parameter $\widetilde{\gamma}(\mathrm{a})$, initial volatility $\widetilde{\omega}(\mathrm{b})$ and correlation $\widetilde{\rho}_{y, \sigma}$ (c) on the shape of the implied volatility smile, $y_{0}=2, \beta=0.5, T_{i}=1$.

In model (3.1) and (3.2) the vol--vol parameter $\widetilde{\gamma}$ mainly accounts for curvature (Rebonato et al. 2011). A second-order effect is that a higher vol-vol value results in a higher smile level. This effect may be negligible, but for the given set of parameter values $^{i}$ it is significant. Further, $\widetilde{\gamma}$ slightly affects skew. The effects are displayed in the left-hand graph in Fig. 1.

The initial volatility $\widetilde{\omega}$ mainly affects the smile level, as can be observed in the graph in the middle in Fig. 1. On the base of an approximation formula, Hagan et al. (2002) discuss that the ATM level of the implied volatility smile traverses along the backbone $\widetilde{\omega} /\left(\bar{y}_{0}^{T_{i}}\right)^{1-\beta}$. The initial volatility has a secondary, but marginal skew effect (Rebonato et al. 2011).

Correlation $\widetilde{\rho}_{y, \sigma}$ also has two effects. It primarily affects the skew, as we see in the right-hand graph of Fig. 1. Hagan et al. (2002) quantify this effect as $\frac{1}{2} \widetilde{\rho}_{y, \sigma} \lambda \log \left(K / \bar{y}_{0}^{T_{i}}\right)$, which they refer to as "vanna skew", with $\lambda=(\widetilde{\gamma} / \widetilde{\omega})\left(\bar{y}_{0}^{T_{i}}\right)^{1-\beta}$ measuring the "strength" of the vol-vol parameter $\widetilde{\gamma}$ compared to the local volatility $\widetilde{\omega} /\left(\bar{y}_{0}^{T_{i}}\right)^{1-\beta}$. A second-order effect of correlation is curvature adjustment: A more negative value for $\widetilde{\rho}_{y, \sigma}$ yields a decrease in curvature. The effect of the skew parameter $\beta$ is three-fold. We first mention its skew effect. A smaller value for $\beta$ implies a more negative skew (a "steeper downward slope" of the implied volatility smile). This effect is most clearly visible for initial forward equal to 1 , see the graph in the middle of Fig. 2. Hagan et al. (2002) determine $-\frac{1}{2}(1-\beta) \log \left(K / \bar{y}_{0}^{T_{i}}\right)$ as being the skew implied by the skew parameter. Second, for an initial forward smaller than 1, a higher $\beta$ value implies a downward shifting, whereas for initial forward larger than 1 this effect is opposite (left-hand graph and right-hand graph of Fig. 2,

${ }^{\mathrm{i}}$ In all figures the parameter values, if not varying, are: $y_{0}=2, \beta=0.5, \widetilde{\gamma}=0.5, \widetilde{\omega}=0.1, \widetilde{\rho}_{y, \sigma}=0$ and $T_{i}=1$. 


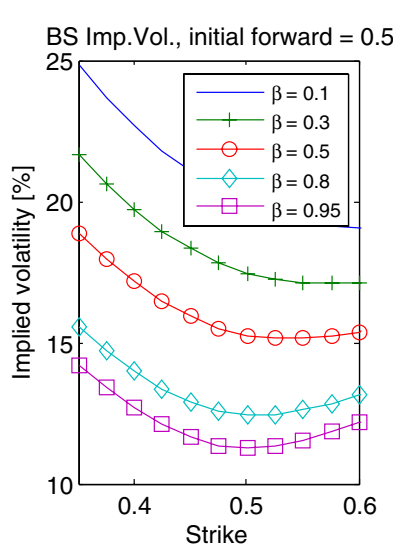

(a)

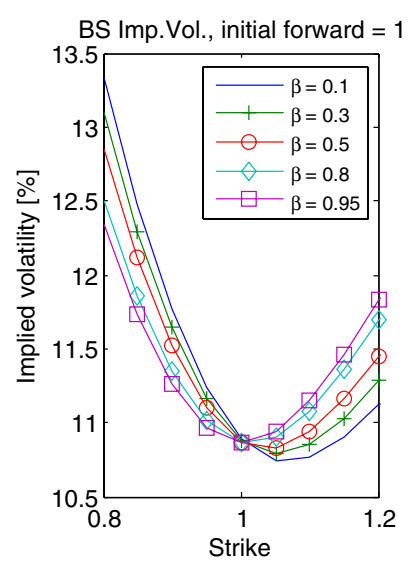

(b)

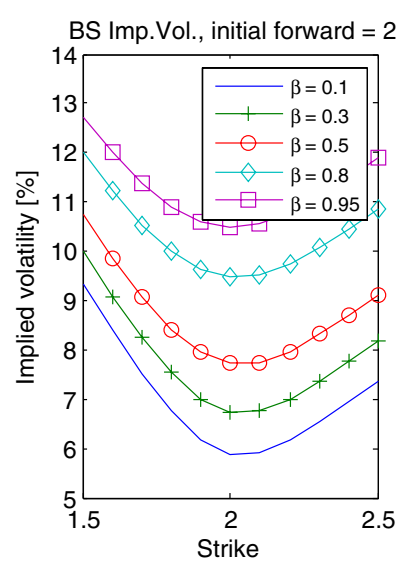

(c)

Fig. 2. Effect of $\beta$ on the shape of the implied volatility smile for initial forward values $\bar{y}_{0}^{T_{i}}=0.5$ (a), $\bar{y}_{0}^{T_{i}}=1(\mathrm{~b})$ and $\bar{y}_{0}^{T_{i}}=2(\mathrm{c}), \widetilde{\gamma}=0.5, \widetilde{\omega}=0.1, \widetilde{\rho}_{y, \sigma}=0, T_{i}=1$.

respectively). This effect is quantified by the backbone $\widetilde{\omega} /\left(\bar{y}_{0}^{T_{i}}\right)^{1-\beta}$. Last, $\beta$ has a third-order curvature effect (Rebonato et al. 2011). As we have defined model (3.1) and (3.2) such that $\bar{y}_{0}^{T_{i}}=1$, Fig. 2(b) applies.

Based on the smile effects just described, we derive effective parameters. We subsequently use the effective model (3.1) and (3.2) to calibrate the time-dependent parameters $\gamma(t), \omega(t)$ and $\rho_{y, \sigma}(t)$. It will turn out that calibrating $\gamma(t)$ and $\rho_{y, \sigma}(t)$ yields a fit to the market in both curvature and skew. What remains, is a mismatch in level, which is compensated for by calibrating $\omega(t)$. We will add a local volatility component on top of the calibrated time-dependent SABR model to compensate for possible calibration inaccuracies.

\subsection{Calibration setup}

The difficulty of calibrating time-dependent parameters lies in the following. It is market practice to calibrate model (3.1) and (3.2) for a grid of - say $N$ expiries. The $N$ parameter values obtained hold from $t=0$ up to the corresponding expiries. In the case of a time-dependent parameter though, we are interested in calibrating one time-dependent function that is consistent with market prices at all $(N)$ expiries.

Let us elaborate on this problem. By means of calibrating the effective model (3.1) and (3.2) in the common way we find, amongst others, vol-vol values $\widetilde{\gamma}_{1}^{\text {mar }}, \widetilde{\gamma}_{2}^{\text {mar }}, \ldots, \widetilde{\gamma}_{N}^{\text {mar }}$ that correspond to the time-intervals $\left[0, T_{1}\right],\left[0, T_{2}\right], \ldots,\left[0, T_{N}\right]$, respectively. We call these "market effective" parameters. Intuitively, $\widetilde{\gamma}_{i}^{\text {mar }}$ "captures" all information up to $T_{i}$. We can also extract market effective term structure values $\widetilde{\omega}_{1}^{\operatorname{mar}}, \widetilde{\omega}_{2}^{\operatorname{mar}}, \ldots, \widetilde{\omega}_{N}^{\operatorname{mar}}$ and correlations $\widetilde{\rho}_{y, \sigma, 1}^{\operatorname{mar}}, \widetilde{\rho}_{y, \sigma, 2}^{\operatorname{mar}}, \ldots, \widetilde{\rho}_{y, \sigma, N}^{\operatorname{mar}}$. In the following, we only describe how the time-dependent vol-vol parameter $\gamma(t)$ is obtained. The same procedure holds for finding $\omega(t)$ and $\rho_{y, \sigma}(t)$. 
The time-dependent vol-vol parameter $\gamma(t)$ has to satisfy two requirements. The first one is trivial: Our goal is to determine one time-dependent function. Second, it should relate to all expiries $T_{i}, i=1,2, \ldots, N$ given in the market. That is, at the given expiries the value of the time-dependent parameter $\gamma\left(T_{i}\right)$ must yield the same implied volatility as the market effective parameter $\widetilde{\gamma}_{i}^{\text {mar }}$ does.

If only the latter requirement had to be satisfied, we could just choose $\gamma\left(T_{i}\right)=$ $\widetilde{\gamma}_{i}^{\text {mar }}$ for all expiries. In this case, we would have $N$ constant functions $\gamma(\cdot)$ that "live" on different time-intervals. The first requirement though complicates the problem of finding a time-dependent parameter: Mapping the set of market effective parameters $\left\{\widetilde{\gamma}_{1}^{\mathrm{mar}}, \widetilde{\gamma}_{2}^{\mathrm{mar}}, \ldots, \widetilde{\gamma}_{N}^{\mathrm{mar}}\right\}$ onto one time-dependent parameter $\gamma(t)$ is not straightforward. An easier problem is to transfer from a time-dependent parameter to its "effective equivalent", so to find a mapping

$$
\left\{\gamma(t), 0 \leq t \leq T_{N}\right\} \rightarrow\left\{\widetilde{\gamma}_{1}^{\bmod }, \widetilde{\gamma}_{2}^{\bmod }, \ldots, \widetilde{\gamma}_{N}^{\bmod }\right\}
$$

where $\widetilde{\gamma}_{i}^{\text {mod }}, i=1,2, \ldots, N$ denote the effective vol-vol parameter values implied by the model, in particular by the time-dependent vol-vol parameter.

Suppose we have established mapping (3.4). Subsequently, in the calibration, we numerically find $\gamma(t)$ such that $\widetilde{\gamma}_{i}^{\text {mod }}=\widetilde{\gamma}_{i}^{\text {mar }}, i=1, \ldots, N$ or on the base of matching implied volatilities obtained using $\widetilde{\gamma}_{i}^{\text {mod }}$ and $\widetilde{\gamma}_{i}^{\text {mar }}$, respectively. From a computational point of view, finding $\gamma(t)$ in this way is significantly less expensive than repeatedly applying a pricing method in the calibration that is suitable for time-dependent parameters (Piterbarg 2005) (e.g. Monte Carlo simulation or a PDE-based approach). In a similar way we find $\omega(t)$ and $\rho_{y, \sigma}(t)$.

\section{Effective Parameters}

Based on their effects on the shape of the implied volatility smile, we derive effective parameters. In the following subsections, we derive mappings for the effective volvol parameter, term structure and correlation, respectively. The results obtained are used in the calibration in Sec. 5 .

\subsection{Effective vol-vol parameter}

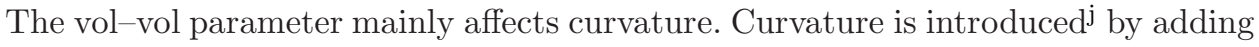
stochastic volatility to the CEV model (Cox 1975), which results in the SABR model. An appropriate measure for curvature is the "realized volatility", which is defined as

$$
\int_{0}^{T_{i}} \omega_{1}(t) \sigma(t) \mathrm{d} W^{T_{i}}(t),
$$

where $\omega_{1}(t)$ is a deterministic scaling parameter specified by $(2.5)$.

jNeglecting the higher-order curvature effects of the correlation and skew parameter. 
We determine the effective vol-vol parameter such that the realized volatilities of the time-dependent and effective models are equal in distribution, that is

$$
\int_{0}^{T_{i}} \omega_{1}(t) \sigma(t) \mathrm{d} W^{T_{i}}(t) \stackrel{d}{=} \int_{0}^{T_{i}} \widetilde{\omega}_{1} \widetilde{\sigma}(t) \mathrm{d} W^{T_{i}}(t) .
$$

More concretely, we obtain the effective vol-vol parameter by matching moments of the realized volatilities.

In the following lemma, we first state the main result of this section. We subsequently provide a proof for it.

Lemma 4.1 (Effective vol-vol parameter). By matching moments of the realized volatilities of the time-dependent and effective models, the effective vol-vol parameter $\tilde{\gamma}$ corresponding to the expiry $T_{i}$ is obtained by the following equation:

$$
\begin{aligned}
& \int_{0}^{T_{i}} \omega_{1}^{2}(t)\left(\int_{0}^{t} \omega_{1}^{2}(s) \mathrm{e}^{6 \int_{0}^{s} \gamma^{2}(u) \mathrm{d} u+\int_{s}^{t} \gamma^{2}(u) \mathrm{d} u} \mathrm{~d} s\right) \mathrm{d} t \\
& \quad=\frac{1}{5}\left(\frac{\int_{0}^{T_{i}} \omega_{1}^{2}(t) \mathrm{e}^{\int_{0}^{t} \gamma^{2}(u) \mathrm{d} u} \mathrm{~d} t}{\mathrm{e}^{\widetilde{\gamma}^{2} T_{i}}-1}\right)^{2}\left(\frac{1}{6} \mathrm{e}^{6 \widetilde{\gamma}^{2} T_{i}}-\mathrm{e}^{\widetilde{\gamma}^{2} T_{i}}+\frac{5}{6}\right) .
\end{aligned}
$$

Proof. It is easy to see that first moment matching of the realized volatilities does not give conclusive results: Trivially, $\mathbb{E}\left[\int_{0}^{T_{i}} \omega_{1}(t) \sigma(t) \mathrm{d} W^{T_{i}}(t)\right]=$ $\mathbb{E}\left[\int_{0}^{T_{i}} \widetilde{\omega}_{1} \widetilde{\sigma}(t) \mathrm{d} W^{T_{i}}(t)\right]=0$. We therefore proceed by matching the variances of the realized volatilities, i.e.

$$
\begin{gathered}
\mathbb{E}\left[\left(\int_{0}^{T_{i}} \omega_{1}(t) \sigma(t) \mathrm{d} W^{T_{i}}(t)\right)^{2}\right]=\mathbb{E}\left[\left(\int_{0}^{T_{i}} \widetilde{\omega}_{1} \widetilde{\sigma}(t) \mathrm{d} W^{T_{i}}(t)\right)^{2}\right] \\
\Leftrightarrow \int_{0}^{T_{i}} \omega_{1}^{2}(t) \mathbb{E}\left[\sigma^{2}(t)\right] \mathrm{d} t=\widetilde{\omega}_{1}^{2} \int_{0}^{T_{i}} \mathbb{E}\left[\widetilde{\sigma}^{2}(t)\right] \mathrm{d} t
\end{gathered}
$$

which gives

$$
\widetilde{\omega}_{1}^{2}=\frac{\int_{0}^{T_{i}} \omega_{1}^{2}(t) \mathbb{E}\left[\sigma^{2}(t)\right] \mathrm{d} t}{\int_{0}^{T_{i}} \mathbb{E}\left[\widetilde{\sigma}^{2}(t)\right] \mathrm{d} t}
$$

As

$$
\mathbb{E}\left[\sigma^{2}(t)\right]=\mathrm{e}^{\int_{0}^{t} \gamma^{2}(u) \mathrm{d} u}, \quad \mathbb{E}\left[\widetilde{\sigma}^{2}(t)\right]=\mathrm{e}^{\widetilde{\gamma}^{2} t},
$$

we have

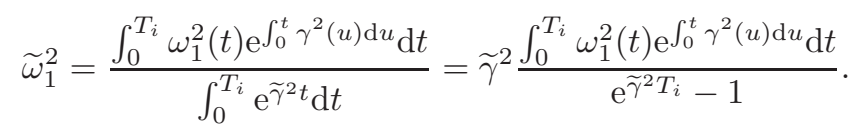

Equation (4.1) yields two unknowns: $\widetilde{\omega}_{1}$ and $\widetilde{\gamma}$. In order to have a system with two equations and two unknowns that we can solve for $\widetilde{\gamma}$, we match another higher 
moment as follows:

$$
\mathbb{E}\left[\left(\int_{0}^{T_{i}} \omega_{1}^{2}(t) \sigma^{2}(t) \mathrm{d} t\right)^{2}\right]=\widetilde{\omega}_{1}^{4} \mathbb{E}\left[\left(\int_{0}^{T_{i}} \tilde{\sigma}^{2}(t) \mathrm{d} t\right)^{2}\right] .
$$

Evaluating the left-hand side of (4.2) gives:

$$
\mathbb{E}\left[\left(\int_{0}^{T_{i}} \omega_{1}^{2}(t) \sigma^{2}(t) \mathrm{d} t\right)^{2}\right]=\mathbb{E}\left[\int_{0}^{T_{i}}\left(\int_{0}^{T_{i}} \omega_{1}^{2}(s) \sigma^{2}(s) \mathrm{d} s\right) \omega_{1}^{2}(t) \sigma^{2}(t) \mathrm{d} t\right] .
$$

By symmetry around the point $s=t$, we have

$$
\begin{aligned}
\mathbb{E}\left[\left(\int_{0}^{T_{i}} \omega_{1}^{2}(t) \sigma^{2}(t) \mathrm{d} t\right)^{2}\right] & =2 \mathbb{E}\left[\int_{0}^{T_{i}} \int_{0}^{t} \omega_{1}^{2}(s) \sigma^{2}(s) \omega_{1}^{2}(t) \sigma^{2}(t) \mathrm{d} s \mathrm{~d} t\right] \\
& =2 \int_{0}^{T_{i}} \omega_{1}^{2}(t) \int_{0}^{t} \omega_{1}^{2}(s) \mathbb{E}\left[\sigma^{2}(s) \sigma^{2}(t)\right] \mathrm{d} s \mathrm{~d} t .
\end{aligned}
$$

To evaluate $\mathbb{E}\left[\sigma^{2}(s) \sigma^{2}(t)\right]$, we use the dynamics of the squared volatility $\sigma^{2}(t)$, which can easily be derived:

$$
\mathrm{d} \sigma^{2}(t)=\sigma^{2}(t)\left(\gamma^{2}(t) \mathrm{d} t+2 \gamma(t) \mathrm{d} W_{\sigma}^{T_{i}}(t)\right) .
$$

Applying basic Itô calculus yields

$$
\mathbb{E}\left[\sigma^{2}(s) \sigma^{2}(t)\right]=\mathrm{e}^{6 \int_{0}^{s} \gamma^{2}(u) \mathrm{d} u+\int_{s}^{t} \gamma^{2}(u) \mathrm{d} u} .
$$

Combining this result with (4.3) gives for the left-hand side of (4.2):

$$
\begin{aligned}
& \mathbb{E}\left[\left(\int_{0}^{T_{i}} \omega_{1}^{2}(t) \sigma^{2}(t) \mathrm{d} t\right)^{2}\right] \\
& \quad=2 \int_{0}^{T_{i}} \omega_{1}^{2}(t)\left(\int_{0}^{t} \omega_{1}^{2}(s) \mathrm{e}^{6 \int_{0}^{s} \gamma^{2}(u) \mathrm{d} u+\int_{s}^{t} \gamma^{2}(u) \mathrm{d} u} \mathrm{~d} s\right) \mathrm{d} t .
\end{aligned}
$$

In a similar way we obtain for the right-hand side

$$
\widetilde{\omega}_{1}^{4} \mathbb{E}\left[\left(\int_{0}^{T_{i}} \widetilde{\sigma}^{2}(t) \mathrm{d} t\right)^{2}\right]=\frac{2 \widetilde{\omega}_{1}^{4}}{5 \widetilde{\gamma}^{4}}\left(\frac{1}{6} \mathrm{e}^{6 \widetilde{\gamma}^{2} T_{i}}-\mathrm{e}^{\widetilde{\gamma}^{2} T_{i}}+\frac{5}{6}\right) .
$$

We now substitute (4.1) in the right-hand side of (4.5) and Eq. (4.2) becomes:

$$
\begin{aligned}
& \int_{0}^{T_{i}} \omega_{1}^{2}(t)\left(\int_{0}^{t} \omega_{1}^{2}(s) \mathrm{e}^{6 \int_{0}^{s} \gamma^{2}(u) \mathrm{d} u+\int_{s}^{t} \gamma^{2}(u) \mathrm{d} u} \mathrm{~d} s\right) \mathrm{d} t \\
& \quad=\frac{1}{5}\left(\frac{\int_{0}^{T_{i}} \omega_{1}^{2}(t) \mathrm{e}^{\int_{0}^{t} \gamma^{2}(u) \mathrm{d} u} \mathrm{~d} t}{\mathrm{e}^{\widetilde{\gamma}^{2} T_{i}}-1}\right)^{2}\left(\frac{1}{6} \mathrm{e}^{6 \widetilde{\gamma}^{2} T_{i}}-\mathrm{e}^{\widetilde{\gamma}^{2} T_{i}}+\frac{5}{6}\right) .
\end{aligned}
$$

We numerically solve this equation for $\widetilde{\gamma}$. The other unknown parameter, $\widetilde{\omega}_{1}$, has vanished. 
Remark 4.1 (Piecewise-constant parameters). In the case of piecewiseconstant parameters we can derive analytical expressions for the integrals in (4.1) and (4.4), which significantly speeds up the calibration procedure.

\subsubsection{Numerical experiment}

Let $y_{0}=2, \beta=0.5, \omega(t)=0.15, r_{d}=0.05$ and $r_{f}=0.02$. Further, we assume $\rho_{y, \sigma}(t)=0$.

Remark 4.2 (Interest rates). In the calibration "effective" and time-dependent domestic and foreign interest rates can be extracted from the initial (non-scaled) forward $y_{0}^{T_{i}}$ and spot $y_{0}$ via the relation $y_{0}^{T_{i}}:=y_{0} \frac{P_{f}\left(0, T_{i}\right)}{P_{d}\left(0, T_{i}\right)}$.

Remark 4.3 (Zero correlation). In the numerical experiments in this section and Sec. 4.2.1, we choose the correlation to be zero. Assuming zero correlation yields the advantage that the exact zero-correlation pricing formula of Antonov et al. (2013) can serve as a benchmark. In the Monte Carlo simulation, we apply a basic first-order Taylor approximation scheme. Typically, Monte Carlo simulation schemes of the SABR model are biased, especially for large vol-vol values and small initial forwards (Chen et al. 2012). In order to make sure that results are not affected by the Monte Carlo bias, we can use the effective parameters both in a Monte Carlo simulation and in the zero-correlation pricing formula (Antonov et al. 2013) to price European call options. In the numerical experiments in this section and Secs. 4.2.1 and 4.3.1 we have confirmed that results are free of Monte Carlo bias. We have also verified that for non-zero correlation values, the performance of the effective parameters is similar to the performance as shown in this section and Sec. 4.2.1.

We assume values for $\gamma(t)$ as given in Table 1 . We choose the vol-vol parameter to be decreasing over time, as in the FX market the curvature of the implied volatility smile typically diminishes for longer expiries. Effective vol-vol values are also given in Table 1. In Fig. 3 we display Black-Scholes implied volatility smiles obtained by simulating the time-dependent model (2.1) and (2.2) and the effective model (also in spot measure) for $T=1, T=2$ and $T=5$ with corresponding $\widetilde{\gamma}$ values (number of paths is $5 \cdot 10^{5}$, number of time-steps per year is 200). The curvature fit is highly satisfactory. ${ }^{\mathrm{k}}$

Table 1. Time-dependent and effective vol-vol values.

\begin{tabular}{cccccc}
\hline$t$ & {$\left[0, \frac{1}{2}\right)$} & {$\left[\frac{1}{2}, 1\right)$} & {$[1,2)$} & {$[2,3)$} & {$[3,5]$} \\
$\gamma(t)$ & 1 & 0.8 & 0.5 & 0.3 & 0.2 \\
$t$ & $\frac{1}{2}$ & 1 & 2 & 3 & 5 \\
$\widetilde{\gamma}$ & 1 & 0.911 & 0.785 & 0.692 & 0.565 \\
\hline
\end{tabular}

\footnotetext{
kWe have derived $\widetilde{\gamma}$ by only considering the vol-vol's primary effect (which is on the smile's curvature). Due to this, a marginal level mismatch occurs between the time-dependent model
} 


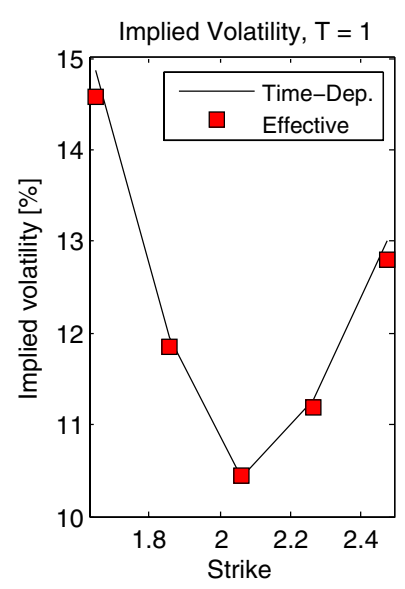

(a)

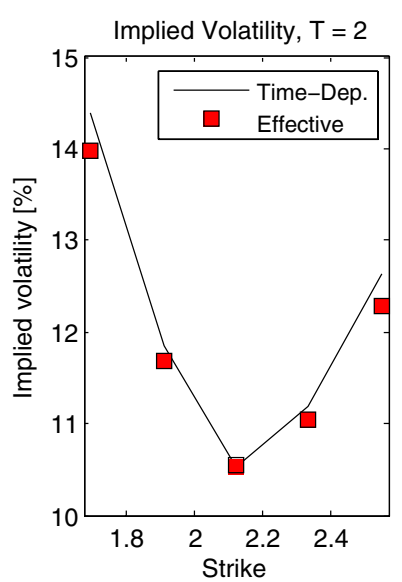

(b)

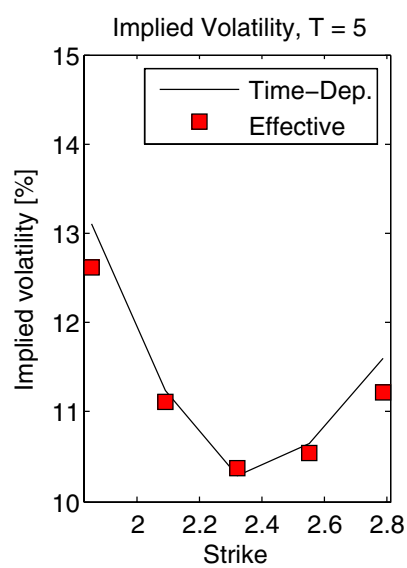

(c)

Fig. 3. Performance of the effective vol-vol parameter for $T=1$ (a), $T=2$ (b) and $T=5$ (c).

\subsection{Effective term structure}

In Sec. 3, we mentioned that the parameter $\omega$ mainly affects the level of the implied volatility smile. Therefore, in this section, we derive the effective term structure $\widetilde{\omega}$ by matching "smile levels" of the time-dependent and effective models. As the level of the smile is completely determined by the ATM implied volatility value, we match ATM prices of the time-dependent and effective models, that is:

$$
\mathbb{E}\left[\left(y^{T_{i}}\left(T_{i}\right)-\bar{y}_{0}^{T_{i}}\right)^{+}\right]=\mathbb{E}\left[\left(\widetilde{y}^{T_{i}}\left(T_{i}\right)-\bar{y}_{0}^{T_{i}}\right)^{+}\right], \quad \bar{y}_{0}^{T_{i}}=1 .
$$

Lemma 4.2. Suppose we assume in SDE system (2.3) and (2.4) for the forward $y^{T_{i}}$ lognormal dynamics. The expected ATM payoff at time $T_{i}$ is given by

$$
\mathbb{E}\left[\left(y^{T_{i}}\left(T_{i}\right)-1\right)^{+}\right]=\mathbb{E}[g(x)],
$$

with

$$
g(x):=2 \Phi\left(\frac{1}{2} \sqrt{x}\right)-1, \quad x:=\int_{0}^{T_{i}} \omega_{1}^{2}(t) \sigma^{2}(t) \mathrm{d} t,
$$

where $\Phi$ denotes the standard normal cumulative distribution function.

Proof. Assuming lognormal dynamics for the forward we have

$$
y^{T_{i}}\left(T_{i}\right)=\mathrm{e}^{-\frac{1}{2} \int_{0}^{T_{i}} \omega_{1}^{2}(t) \sigma^{2}(t) \mathrm{d} t+\int_{0}^{T_{i}} \omega_{1}(t) \sigma(t) \mathrm{d} W_{y}^{T_{i}}(t)} .
$$

and the effective model, as the vol-vol parameter also has a level effect. In order to obtain a level fit again, we adjust the term structure value of the effective model slightly by exploiting the techniques of Sec. 4.2. 
Suppose that the path $\left\{\omega_{1}(t) \sigma(t), 0 \leq t \leq T_{i}\right\}$ is given. Defining

$$
Z\left(T_{i}\right):=\int_{0}^{T_{i}} \omega_{1}^{2}(t) \sigma^{2}(t) \mathrm{d} t, \quad \tilde{\sigma}^{2}:=\frac{Z\left(T_{i}\right)}{T_{i}},
$$

we have

$$
y^{T_{i}}\left(T_{i}\right)=\mathrm{e}^{-\frac{1}{2} \widetilde{\sigma}^{2} T_{i}+\widetilde{\sigma} W_{y}^{T_{i}}\left(T_{i}\right)}
$$

and also

$$
\mathbb{E}\left[\left(y^{T_{i}}\left(T_{i}\right)-1\right)^{+} \mid \omega_{1}(t) \sigma(t), 0 \leq t \leq T_{i}\right]=\Phi\left(d_{+}\right)-\Phi\left(d_{-}\right),
$$

with

$$
d_{ \pm}=\frac{\log (1) \pm \frac{1}{2} \widetilde{\sigma}^{2} T_{i}}{\widetilde{\sigma} \sqrt{T_{i}}}= \pm \frac{1}{2} \widetilde{\sigma} \sqrt{T_{i}}= \pm \frac{1}{2} \sqrt{Z\left(T_{i}\right)}
$$

As a consequence, the conditional expectation can be written as:

$$
\begin{aligned}
\mathbb{E}\left[\left(y^{T_{i}}\left(T_{i}\right)-1\right)^{+} \mid \omega_{1}(t) \sigma(t), 0 \leq t \leq T_{i}\right] & =\Phi\left(\frac{1}{2} \sqrt{Z\left(T_{i}\right)}\right)-\Phi\left(-\frac{1}{2} \sqrt{Z\left(T_{i}\right)}\right) \\
& =2 \Phi\left(\frac{1}{2} \sqrt{Z\left(T_{i}\right)}\right)-1 .
\end{aligned}
$$

Applying the Tower property yields:

$$
\begin{aligned}
\mathbb{E}\left[\left(y^{T_{i}}\left(T_{i}\right)-1\right)^{+}\right] & =\mathbb{E}\left[\mathbb{E}\left[\left(y^{T_{i}}\left(T_{i}\right)-1\right)^{+} \mid \omega_{1}(t) \sigma(t), 0 \leq t \leq T_{i}\right]\right] \\
& =\mathbb{E}[g(x)]
\end{aligned}
$$

with

$$
g(x):=2 \Phi\left(\frac{1}{2} \sqrt{x}\right)-1, \quad x:=\int_{0}^{T_{i}} \omega_{1}^{2}(t) \sigma^{2}(t) \mathrm{d} t .
$$

Analogously, for the effective model we have $\mathbb{E}\left[\left(\widetilde{y}^{T_{i}}\left(T_{i}\right)-1\right)^{+}\right]=\mathbb{E}[g(\widetilde{x})]$ with $\widetilde{x}:=\widetilde{\omega}_{1}^{2} \int_{0}^{T_{i}} \tilde{\sigma}^{2}(t) \mathrm{d} t$. We continue with approximating $g(\cdot)$ by a simpler function, which yields efficient evaluation of the effective term structure.

Remark 4.4. In this section, we use the superscripts $(\ell)$ and $(r)$ to indicate approximation errors corresponding to the left-hand side and right-hand side of Eq. (4.6), respectively.

Lemma 4.3. Approximating $g(\cdot)$ by the corresponding Taylor series yields

$$
\mathbb{E}\left[\left(y^{T_{i}}\left(T_{i}\right)-1\right)^{+}\right]=\frac{1}{\sqrt{2 \pi}} \mathbb{E}[\sqrt{x}]+\epsilon_{\mathrm{T}}^{(\ell)},
$$

with

$$
\epsilon_{\mathrm{T}}^{(\ell)}:=\frac{2}{\sqrt{\pi}}\left(-\frac{1}{3} \mathbb{E}\left[z^{3}\right]+\frac{1}{10} \mathbb{E}\left[z^{5}\right]-\cdots\right), \quad z:=\frac{1}{2} \sqrt{x / 2}, \quad x:=\int_{0}^{T_{i}} \omega_{1}^{2}(t) \sigma^{2}(t) \mathrm{d} t .
$$


Proof. By definition we have

$$
\Phi\left(\frac{1}{2} \sqrt{x}\right)=\frac{1}{2}\left[1+\operatorname{erf}\left(\frac{1}{2} \sqrt{x / 2}\right)\right],
$$

where $\operatorname{erf}(\cdot)$ denotes the (Gauss) error function. ${ }^{1}$ Defining

$$
x:=\int_{0}^{T_{i}} \omega_{1}^{2}(t) \sigma^{2}(t) \mathrm{d} t, \quad z:=\frac{1}{2} \sqrt{x / 2},
$$

we approximate the integrand of the error function by the first term of the corresponding Taylor series around 0:

$$
\operatorname{erf}(z)=\frac{2}{\sqrt{\pi}} \int_{0}^{z} \frac{\left(-t^{2}\right)^{0}}{0 !} \mathrm{d} t+\bar{\epsilon}_{\mathrm{T}}^{(\ell)}=\frac{2}{\sqrt{\pi}} z+\bar{\epsilon}_{\mathrm{T}}^{(\ell)},
$$

with

$$
\bar{\epsilon}_{\mathrm{T}}^{(\ell)}:=\frac{2}{\sqrt{\pi}}\left(-\frac{1}{3} z^{3}+\frac{1}{10} z^{5}-\cdots\right),
$$

denoting the truncation error. Combining (4.8) and (4.9) we obtain

$$
g(x)=\frac{1}{\sqrt{2 \pi}} \sqrt{x}+\bar{\epsilon}_{\mathrm{T}}^{(\ell)}
$$

and thus

$$
\mathbb{E}\left[\left(y^{T_{i}}\left(T_{i}\right)-1\right)^{+}\right]=\mathbb{E}[g(x)]=\frac{1}{\sqrt{2 \pi}} \mathbb{E}[\sqrt{x}]+\epsilon_{\mathrm{T}}^{(\ell)},
$$

with

$$
\epsilon_{\mathrm{T}}^{(\ell)}:=\frac{2}{\sqrt{\pi}}\left(-\frac{1}{3} \mathbb{E}\left[z^{3}\right]+\frac{1}{10} \mathbb{E}\left[z^{5}\right]-\cdots\right) .
$$

Remark 4.5 (Qualitative analysis of $\epsilon_{\mathbf{T}}^{(\ell)}$ ). The truncation error $\epsilon_{\mathrm{T}}^{(\ell)}$ is quantified as follows. We distinguish between two cases, namely $\frac{P_{d}\left(T_{i}, T_{N}\right)}{y_{0}^{T_{i}} P_{f}\left(T_{i}, T_{N}\right)} \leq 1$ and $\frac{P_{d}\left(T_{i}, T_{N}\right)}{y_{0}^{T_{i}} P_{f}\left(T_{i}, T_{N}\right)}>1$. For the former case, by definition of $\omega_{1}(\cdot)$, the truncation error is largest for $\beta=1$. Typically $\omega(\cdot)=\mathcal{O}\left(10^{-1}\right)$ and as $\beta=1$, we have $\omega_{1}(\cdot)=\omega(\cdot)=\mathcal{O}\left(10^{-1}\right)$. Further, as the scaled volatility $\sigma(\cdot)$ has initial value 1 , $\mathbb{E}[z]=\mathcal{O}\left(10^{-1}\right)$. For realistic values for the vol-vol parameter, the leading term in the truncation error is $\mathbb{E}\left[z^{3}\right]$ and is assumed to have smaller order of magnitude than $\mathbb{E}[z]$. For $\beta<1$, the truncation error is smaller. In case $\frac{P_{d}\left(T_{i}, T_{N}\right)}{y_{0}^{T_{i}} P_{f}\left(T_{i}, T_{N}\right)}>1$, the truncation error is largest for $\beta=0$, and $\omega(\cdot)=\mathcal{O}\left(10^{-1}\right)$ results in $\omega_{1}^{2}(\cdot)=\mathcal{O}\left(\left(10 y_{0}^{T_{i}}\right)^{-2}\right)$. As the scaled volatility $\sigma(\cdot)$ has initial value 1 , typically $\mathbb{E}[z]=\mathcal{O}\left(\left(10 y_{0}^{T_{i}}\right)^{-1}\right)$. Realistic vol-vol values imply that $\mathbb{E}\left[z^{3}\right]$ is the leading term in the truncation error.

${ }^{1}$ The Gauss error function is defined as

$$
\operatorname{erf}(x):=\frac{2}{\sqrt{\pi}} \int_{0}^{x} \mathrm{e}^{-t^{2}} \mathrm{~d} t .
$$


A smaller $y_{0}^{T_{i}}$ value implies a larger truncation error and the contribution of the non-parametric local volatility compensator is more significant. For $\beta>0$ the truncation error is smaller. In the numerical experiments in Sec. 4.2.1, we show that relatively large $\omega(\cdot)$ and $\gamma(\cdot)$ values imply a slightly less accurate level fit, which is in line with the former qualitative analysis.

Analogous to the time-dependent model, the expected ATM payoff corresponding to the effective model is given by

$$
\mathbb{E}\left[\left(\widetilde{y}^{T_{i}}\left(T_{i}\right)-1\right)^{+}\right]=\frac{1}{\sqrt{2 \pi}} \mathbb{E}[\sqrt{\widetilde{x}}]+\epsilon_{\mathrm{T}}^{(r)},
$$

with

$$
\epsilon_{\mathrm{T}}^{(r)}:=\frac{2}{\sqrt{\pi}}\left(-\frac{1}{3} \mathbb{E}\left[\widetilde{z}^{3}\right]+\frac{1}{10} \mathbb{E}\left[\widetilde{z}^{5}\right]-\cdots\right), \quad \widetilde{z}:=\frac{1}{2} \sqrt{\widetilde{x} / 2}, \quad \widetilde{x}:=\widetilde{\omega}_{1}^{2} \int_{0}^{T_{i}} \widetilde{\sigma}^{2}(t) \mathrm{d} t .
$$

For the truncation error $\epsilon_{\mathrm{T}}^{(r)}$, a similar analysis holds as in Remark 4.5. Substitution of (4.7) and (4.10) in Eq. (4.6) gives

$$
\frac{1}{\sqrt{2 \pi}} \mathbb{E}\left[\sqrt{\int_{0}^{T_{i}} \omega_{1}^{2}(t) \sigma^{2}(t) \mathrm{d} t}\right]+\epsilon_{\mathrm{T}}^{(\ell)}=\frac{\widetilde{\omega}_{1}}{\sqrt{2 \pi}} \mathbb{E}\left[\sqrt{\int_{0}^{T_{i}} \tilde{\sigma}^{2}(t) \mathrm{d} t}\right]+\epsilon_{\mathrm{T}}^{(r)} .
$$

In order to evaluate the expectations, we derive closed-form expressions for the " $\frac{1}{2}$ th moments" of the integrals in (4.11).

Lemma 4.4 (Effective term structure). An approximation of the expected ATM payoff corresponding to the time-dependent model in (2.3) and (2.4) is given by

$$
\mathbb{E}\left[\left(y^{T_{i}}\left(T_{i}\right)-1\right)^{+}\right]=\frac{1}{\sqrt{2 \pi}}\left(\omega_{1}(0) \sqrt{\Delta_{t}} \widehat{\phi}_{Y_{M}}\left(-\frac{1}{2} i\right)\right)+\epsilon_{\mathrm{T}}^{(\ell)}+\epsilon_{\mathrm{I}}^{(\ell)}+\epsilon_{\mathrm{F}}^{(\ell)},
$$

with $\widehat{\phi}_{Y_{M}}(\cdot)$ denoting an approximation of the characteristic function of

$$
Y_{M}:=\log \left(\sum_{j=1}^{M} \frac{\omega_{1}^{2}\left(t_{j}\right) \sigma^{2}\left(t_{j}\right)}{\omega_{1}^{2}(0)}\right)
$$

with $t_{j}=j \Delta_{t}, \Delta_{t}=T_{i} / M, j=1, \ldots, M$. The error $\epsilon_{\mathrm{I}}^{(\ell)}$ is due to an integral approximation and $\epsilon_{\mathrm{F}}^{(\ell)}$ is introduced in the characteristic function approximation. Further, from (4.12) and a similar result for the effective model, the effective term structure corresponding to the expiry $T_{i}$ is given by

$$
\widetilde{\omega}=\widetilde{\omega}_{1}\left(\frac{y_{0}^{T_{i}} P_{f}\left(T_{i}, T_{N}\right)}{P_{d}\left(T_{i}, T_{N}\right)}\right)^{1-\beta} \quad, \quad \text { with } \widetilde{\omega}_{1}=\frac{\omega_{1}(0) \widehat{\phi}_{Y_{M}}\left(-\frac{1}{2} i\right)}{\widehat{\phi}_{\widetilde{Y}_{M}}\left(-\frac{1}{2} i\right)}+\varepsilon,
$$


where $\widehat{\phi}_{\widetilde{Y}_{M}}(\cdot)$ is an approximation of the characteristic function of

$$
\widetilde{Y}_{M}:=\log \left(\sum_{j=1}^{M} \tilde{\sigma}^{2}\left(t_{j}\right)\right)
$$

and $\varepsilon$ represents the different error terms introduced, which are marginal and do not significantly affect the performance of the effective term structure parameter.

Proof. We start with approximating the integral

$$
\int_{0}^{T_{i}} \omega_{1}^{2}(t) \sigma^{2}(t) \mathrm{d} t=\Delta_{t} \sum_{j=1}^{M} \omega_{1}^{2}\left(t_{j}\right) \sigma^{2}\left(t_{j}\right)+\bar{\epsilon}_{\mathrm{I}}^{(\ell)},
$$

with $\Delta_{t}=T_{i} / M$ and $\bar{\epsilon}_{\mathrm{I}}^{(\ell)}$ denoting the error term corresponding to the time-interval $\left[0, T_{i}\right]$. Given the integral approximation we have

$$
\mathbb{E}\left[\sqrt{\int_{0}^{T_{i}} \omega_{1}^{2}(t) \sigma^{2}(t) \mathrm{d} t}\right]=\omega_{1}(0) \sqrt{\Delta_{t}} \mathbb{E}\left[\left(\sum_{j=1}^{M} \frac{\omega_{1}^{2}\left(t_{j}\right) \sigma^{2}\left(t_{j}\right)}{\omega_{1}^{2}(0)}\right)^{1 / 2}\right]+\epsilon_{\mathrm{I}}^{(\ell)},
$$

with $\Delta_{t}=T_{i} / M$ and $\epsilon_{\mathrm{I}}^{(\ell)}$ is due to the integral approximation error $\bar{\epsilon}_{\mathrm{I}}^{(\ell)}$ in (4.14). We evaluate the expectation as follows. Given the characteristic function $\phi_{Y_{M}}(\cdot)$ of

$$
Y_{M}:=\log \left(\sum_{j=1}^{M} \frac{\omega_{1}^{2}\left(t_{j}\right) \sigma^{2}\left(t_{j}\right)}{\omega_{1}^{2}(0)}\right)
$$

we have

$$
\mathbb{E}\left[\left(\sum_{j=1}^{M} \frac{\omega_{1}^{2}\left(t_{j}\right) \sigma^{2}\left(t_{j}\right)}{\omega_{1}^{2}(0)}\right)^{1 / 2}\right]=\mathbb{E}\left[\mathrm{e}^{\frac{1}{2} Y_{M}}\right]=\phi_{Y_{M}}\left(-\frac{1}{2} i\right)
$$

and thus

$$
\mathbb{E}\left[\sqrt{\int_{0}^{T_{i}} \omega_{1}^{2}(t) \sigma^{2}(t) \mathrm{d} t}\right]=\omega_{1}(0) \sqrt{\Delta_{t}} \widehat{\phi}_{Y_{M}}\left(-\frac{1}{2} i\right)+\epsilon_{\mathrm{I}}^{(\ell)}+\epsilon_{\mathrm{F}}^{(\ell)},
$$

where $\epsilon_{\mathrm{F}}^{(\ell)}$ denotes the error in the approximation of the characteristic function $\widehat{\phi}_{Y_{M}}(\cdot)$, which is introduced in the procedure of recovering it. In Appendix A, we describe the recovery procedure, which was developed in the context of Asian options in Zhang \& Oosterlee (2013). Furthermore, combining (4.17) with the result in (4.7) gives $^{\mathrm{m}}$

$$
\mathbb{E}\left[\left(y^{T_{i}}\left(T_{i}\right)-1\right)^{+}\right]=\frac{1}{\sqrt{2 \pi}}\left(\omega_{1}(0) \sqrt{\Delta_{t}} \widehat{\phi}_{Y_{M}}\left(-\frac{1}{2} i\right)\right)+\epsilon_{\mathrm{T}}^{(\ell)}+\epsilon_{\mathrm{I}}^{(\ell)}+\epsilon_{\mathrm{F}}^{(\ell)} .
$$

${ }^{\mathrm{m}}$ In this step, the error terms $\epsilon_{\mathrm{I}}^{(\ell)}$ and $\epsilon_{\mathrm{F}}^{(\ell)}$ are divided by $\sqrt{2 \pi}$ and we keep the same notation for the "new" error terms. 
For the effective model we can derive

$$
\mathbb{E}\left[\left(\widetilde{y}^{T_{i}}\left(T_{i}\right)-1\right)^{+}\right]=\frac{1}{\sqrt{2 \pi}}\left(\widetilde{\omega}_{1} \sqrt{\Delta_{t}} \widehat{\phi}_{\widetilde{Y}_{M}}\left(-\frac{1}{2} i\right)\right)+\epsilon_{\mathrm{T}}^{(r)}+\epsilon_{\mathrm{I}}^{(r)}+\epsilon_{\mathrm{F}}^{(r)},
$$

where $\widehat{\phi}_{\widetilde{Y}_{M}}(\cdot)$ is an approximation of the characteristic function of

$$
\widetilde{Y}_{M}:=\log \left(\sum_{j=1}^{M} \tilde{\sigma}^{2}\left(t_{j}\right)\right)
$$

Given the identities (4.18) and (4.19), Eq. (4.11) can be written as

$$
\begin{gathered}
\frac{1}{\sqrt{2 \pi}}\left(\omega_{1}(0) \sqrt{\Delta_{t}} \widehat{\phi}_{Y_{M}}\left(-\frac{1}{2} i\right)\right)+\epsilon_{\mathrm{I}}^{(\ell)}+\epsilon_{\mathrm{F}}^{(\ell)}+\epsilon_{\mathrm{T}}^{(\ell)} \\
=\frac{\widetilde{\omega}_{1}}{\sqrt{2 \pi}}\left(\sqrt{\Delta_{t}} \widehat{\phi}_{\widetilde{Y}_{M}}\left(-\frac{1}{2} i\right)\right)+\epsilon_{\mathrm{I}}^{(r)}+\epsilon_{\mathrm{F}}^{(r)}+\epsilon_{\mathrm{T}}^{(r)}
\end{gathered}
$$

and we obtain

$$
\widetilde{\omega}_{1}=\frac{\omega_{1}(0) \widehat{\phi}_{Y_{M}}\left(-\frac{1}{2} i\right)+\epsilon_{\mathrm{I}}^{(\ell)}+\epsilon_{\mathrm{F}}^{(\ell)}+\epsilon_{\mathrm{T}}^{(\ell)}-\epsilon_{\mathrm{T}}^{(r)}}{\widehat{\phi}_{\widetilde{Y}_{M}}\left(-\frac{1}{2} i\right)+\epsilon_{\mathrm{I}}^{(r)}+\epsilon_{\mathrm{F}}^{(r)}}=\frac{\omega_{1}(0) \widehat{\phi}_{Y_{M}}\left(-\frac{1}{2} i\right)}{\widehat{\phi}_{\widetilde{Y}_{M}}\left(-\frac{1}{2} i\right)}+\varepsilon,
$$

where $\varepsilon$ represents the different error terms. By definition (3.3) we arrive at the result in Eq. (4.13).

Lemma 4.5. For the integral approximation error $\epsilon_{\mathrm{I}}^{(\ell)}$ in (4.12) holds: $\lim _{M \rightarrow \infty}\left\|\epsilon_{\mathrm{I}}^{(\ell)}\right\|_{L^{2}}^{2}=0$.

Proof. For the error $\bar{\epsilon}_{\mathrm{I}}^{(\ell)}$ introduced in (4.14) we have:

$$
\begin{aligned}
\mathbb{E}\left|\bar{\epsilon}_{\mathrm{I}}^{(\ell)}\right|^{2} & =\mathbb{E}\left[\left|\int_{0}^{T_{i}} \omega_{1}^{2}(t) \sigma^{2}(t) \mathrm{d} t-\Delta_{t} \sum_{j=1}^{M} \omega_{1}^{2}\left(t_{j}\right) \sigma^{2}\left(t_{j}\right)\right|^{2}\right] \\
& =\mathbb{E}\left[\left|\sum_{j=1}^{M} \int_{t_{j-1}}^{t_{j}}\left\{\omega_{1}^{2}(t) \sigma^{2}(t)-\omega_{1}^{2}\left(t_{j}\right) \sigma^{2}\left(t_{j}\right)\right\} \mathrm{d} t\right|^{2}\right] \\
& \leq \sum_{j=1}^{M} \mathbb{E}\left[\int_{t_{j-1}}^{t_{j}}\left|\omega_{1}^{2}(t) \sigma^{2}(t)-\omega_{1}^{2}\left(t_{j}\right) \sigma^{2}\left(t_{j}\right)\right|^{2} \mathrm{~d} t\right] .
\end{aligned}
$$

Convergence of the integral approximations is evident. An important result in stochastic calculus (see e.g. Karatzas and Shreve 1991, Shreve 2004) states that for each fixed $T>0$ and for any bounded, adapted and measurable process $X(\cdot)$, 
there exists a sequence $\left\{X^{(M)}(\cdot)\right\}_{M=1}^{\infty}$ of simple processes (which are, by definition, bounded $^{\mathrm{n}}$ ) such that

$$
\lim _{M \rightarrow \infty} \mathbb{E}\left[\int_{0}^{T}\left|X^{(M)}(t)-X(t)\right|^{2} \mathrm{~d} t\right]=0 .
$$

In our case, defining $X(t):=\omega_{1}^{2}(t) \sigma^{2}(t)$ and $X^{(M)}(t):=\sum_{j=1}^{M-1} \omega_{1}^{2}\left(t_{j}\right) \sigma^{2}\left(t_{j}\right)$ $\mathbb{1}_{\left\{t_{j-1} \leq t<t_{j}\right\}}(t)+\omega_{1}^{2}\left(t_{M}\right) \sigma^{2}\left(t_{M}\right) \mathbb{1}_{\left\{t_{M-1} \leq t \leq t_{M}\right\}}(t)$ as a simple process approximating $X(t)$ on the interval $\left[0, t_{M}\right]$ with $t_{M}=T_{i}$ (both $X(\cdot)$ and $X^{(M)}(\cdot)$ satisfy the regular conditions), we have:

$$
\begin{aligned}
\lim _{M \rightarrow \infty} \mathbb{E} & {\left[\int_{0}^{T_{i}} \mid \sum_{j=1}^{M-1} \omega_{1}^{2}\left(t_{j}\right) \sigma^{2}\left(t_{j}\right) \mathbb{1}_{\left\{t_{j-1} \leq t<t_{j}\right\}}(t)\right.} \\
& \left.+\omega_{1}^{2}\left(t_{M}\right) \sigma^{2}\left(t_{M}\right) \mathbb{1}_{\left\{t_{M-1} \leq t \leq t_{M}\right\}}(t)-\left.\omega_{1}^{2}(t) \sigma^{2}(t)\right|^{2} \mathrm{~d} t\right]=0 \\
\Leftrightarrow & \lim _{M \rightarrow \infty} \mathbb{E}\left[\sum_{j=1}^{M} \int_{t_{j-1}}^{t_{j}}\left|\omega_{1}^{2}\left(t_{j}\right) \sigma^{2}\left(t_{j}\right)-\omega_{1}^{2}(t) \sigma^{2}(t)\right|^{2} \mathrm{~d} t\right]=0
\end{aligned}
$$

and thus

$$
\lim _{M \rightarrow \infty} \sum_{j=1}^{M} \mathbb{E}\left[\int_{t_{j-1}}^{t_{j}}\left|\omega_{1}^{2}\left(t_{j}\right) \sigma^{2}\left(t_{j}\right)-\omega_{1}^{2}(t) \sigma^{2}(t)\right|^{2} \mathrm{~d} t\right]=0 .
$$

Combining (4.20) and (4.21) yields

$$
\lim _{M \rightarrow \infty}\left\|\bar{\epsilon}_{\mathrm{I}}^{(\ell)}\right\|_{L^{2}}^{2}:=\lim _{M \rightarrow \infty} \mathbb{E}\left|\bar{\epsilon}_{\mathrm{I}}^{(\ell)}\right|^{2}=0
$$

where $\|X\|_{L^{2}}:=\left(\mathbb{E}|X|^{2}\right)^{1 / 2}$ is defined as the norm in $L^{2}$ space. As the error $\epsilon_{\mathrm{I}}^{(\ell)}$ in (4.15) propagates from $\bar{\epsilon}_{\mathrm{I}}^{(\ell)}$, we have $\lim _{M \rightarrow \infty}\left\|\epsilon_{\mathrm{I}}^{(\ell)}\right\|_{L^{2}}^{2}=0$.

Remark 4.6 (Analysis of $\epsilon_{\mathbf{F}}^{(\ell)}$ ). As stated by Zhang \& Oosterlee (2013), three different types of errors are involved in the characteristic function recovery, namely a truncation error $\epsilon_{\mathrm{t}}$, an error of the Fourier cosine expansion $\epsilon_{\mathrm{f}}$ and an error term introduced by applying the Clenshaw-Curtis quadrature $\epsilon_{\mathrm{q}}$. The truncation error is defined as

$$
\epsilon_{\mathrm{t}}\left(Y_{M}\right):=\int_{\mathbb{R} \backslash[a, b]} f_{Y_{M}}(y) \mathrm{d} y
$$

with $f_{Y_{M}}(\cdot)$ denoting the probability density function of $Y_{M}$ defined in (4.16). By definition (4.22), the truncation error decreases as the interval $[a, b]$ increases and the

${ }^{\mathrm{n}}$ Besides for the boundedness, the random variable in each piece of the simple process $-\sigma^{2}\left(t_{j}\right)$ in our case - is $\mathcal{F}\left(t_{j-1}\right)$-measurable. 
error is not dominant for a sufficiently large integration range. Further, from Fang \& Oosterlee (2013) we know that for a probability density function $f(y \mid x) \in \mathbb{C}^{\infty}[a, b]$, the error $\epsilon_{\mathrm{f}}$ of the Fourier cosine expansion is bounded by

$$
\left|\epsilon_{\mathrm{f}}(N,[a, b])\right| \leq R(N) \mathrm{e}^{-(N-1) \xi}
$$

where $\xi>0$ is a constant and the term $R(N)$ is changing less than exponentially with respect to $N$, the number of Fourier cosine terms. So $\epsilon_{\mathrm{f}}$ decays exponentially with respect to $N$, i.e. $\lim _{N \rightarrow \infty} \epsilon_{\mathrm{f}}(N,[a, b])=0$. This error is related to $\epsilon_{\mathrm{F}}^{(\ell)}$, the error in the recovered characteristic function $\widehat{\phi}_{Y_{M}}(\cdot)$, as follows:

$$
\left|\epsilon_{\mathrm{F}}^{(\ell)}\right|=\mathcal{O}\left((M-1)\left(\left|\epsilon_{\mathrm{f}}\right|+\left|\epsilon_{\mathrm{q}}\right|\right)\right) .
$$

Equations (4.23) and (4.24) show that if the number of monitoring dates $M$ increases, we need to increase the number of Fourier expansion terms $N$ to compensate for this and to reach a specified level of accuracy, i.e. $\lim _{M, N \rightarrow \infty} \epsilon_{\mathrm{F}}^{(\ell)}=0$ (neglecting the error $\epsilon_{\mathrm{q}}$ ).

For the effective model, we can also show that for the integral approximation error $\epsilon_{\mathrm{I}}^{(r)}$ in (4.19) $\lim _{M \rightarrow \infty}\left\|\epsilon_{\mathrm{I}}^{(r)}\right\|_{L^{2}}^{2}=0$ and $\lim _{M, N \rightarrow \infty} \epsilon_{\mathrm{F}}^{(r)}=0$.

Remark 4.7 (Level effect). The assumption of lognormal dynamics in (2.3) and (2.4) does not significantly affect the quality of the effective term structure for $\beta \neq 1$. The reason for this is the marginal ATM level effect of the CEV exponent, as we have defined model (2.3) and (2.4) such that $\bar{y}_{0}^{T_{i}}=1$. We could already see this when discussing the smile effects of the skew parameter in Sec. 3. For the effective model, we can quantify the ATM level effect of $\beta$ on the base of Hagan's formula for ATM options (Hagan et al. 2002). Our numerical experiments in Sec. 4.2.1, confirm that the lognormality assumption still yields accurate results when assuming $\beta=$ 0.5. Further, in the calibration procedure we add a non-parametric local volatility component that compensates for possible calibration inaccuracies introduced by the lognormality assumption.

Remark 4.8 (Alternative approaches). We have followed two alternative approaches to find the effective term structure. From the characteristic functions $\widehat{\phi}_{Y_{M}}(\cdot)$ and $\widehat{\phi}_{\widetilde{Y}_{M}}(\cdot)$ the corresponding probability density functions are derived. Successively, we compute approximations of $\mathbb{E}[g(x)]$ and $\mathbb{E}[g(\widetilde{x})]$ with $x$ and $\widetilde{x}$ as defined earlier, without approximating $g(\cdot)$. As a second approach, we have implemented Curran's Asian option pricing method (Curran 1994) and the enhanced "Curran 2M+" method (Deelstra et al. 2004, Lord 2006). From a grid of Asian option prices with underlyings $\sum_{j=1}^{M} \omega_{1}^{2}\left(t_{j}\right) \sigma^{2}\left(t_{j}\right) / M$ and $\sum_{j=1}^{M} \tilde{\sigma}^{2}\left(t_{j}\right) / M$ the corresponding densities are derived by the well-known relation $f\left(K, T ; y_{0}\right)=\partial^{2} C(\cdot) / \partial K^{2}$ (Dupire 1994 ) and we successively calculate approximations of $\mathbb{E}[g(x)]$ and $\mathbb{E}[g(\widetilde{x})]$. In both approaches, $\widetilde{\omega}_{1}$ is determined by an optimization procedure in which $\mathbb{E}[g(x)]$ and $\mathbb{E}[g(\widetilde{x})]$ are repeatedly recalculated. The problem remains though how to efficiently determine the effective term structure value. In contrast, the benefit of Eq. (4.11) 
Table 2. Time-dependent parameter values.

\begin{tabular}{ccccccc}
\hline & $t$ & {$\left[0, \frac{1}{2}\right)$} & {$\left[\frac{1}{2}, 1\right)$} & {$[1,2)$} & {$[2,3)$} & {$[3,5]$} \\
\hline Case I & $\gamma(t)$ & 0.7 & 0.7 & 0.7 & 0.7 & 0.7 \\
& $\omega(t)$ & 0.1 & 0.12 & 0.14 & 0.16 & 0.18 \\
Case II & $\gamma(t)$ & 0.3 & 0.3 & 0.3 & 0.3 & 0.3 \\
& $\omega(t)$ & 0.1 & 0.12 & 0.14 & 0.16 & 0.18 \\
Case III & $\gamma(t)$ & 0.3 & 0.3 & 0.3 & 0.3 & 0.3 \\
& $\omega(t)$ & 0.3 & 0.36 & 0.42 & 0.48 & 0.54 \\
Case IV & $\gamma(t)$ & 1 & 0.8 & 0.5 & 0.3 & 0.2 \\
& $\omega(t)$ & 0.1 & 0.12 & 0.14 & 0.16 & 0.18 \\
\hline
\end{tabular}

is the closed-form approximation of $\widetilde{\omega}_{1}$, which yields efficient evaluation and highly accurate results (see Sec. 4.2.1).

\subsubsection{Numerical experiment}

In this section, we test the performance of the effective term structure $\widetilde{\omega}$ given by (4.13) for four cases. For all experiments $y_{0}=2, \beta=0.5$, domestic and foreign interest rates are $r_{d}=0.05$ and $r_{f}=0.02$, respectively, and $\rho_{y, \sigma}(t)=0$.

We first test the stand-alone performance of $\widetilde{\omega}$ in Cases I, II and III (the Monte Carlo simulation consists of $5 \cdot 10^{5}$ paths and 200 time-steps per year). Timedependent parameter values are given in Table 2 . We only consider the last expiry $T=5$.

In Case I $\gamma(t)$ has a rather extreme value, especially for the larger times to maturity, whereas the $\omega(t)$ value is chosen to be moderate. For Case III the opposite holds. In Case II, both $\gamma(t)$ and $\omega(t)$ have moderate values. In Case IV, we test the combined performance of the effective vol-vol and effective term structure parameters across multiple expiries. In this case, we assume parameter values which we typically observe in FX markets.

The effective parameter values are given in Table 3. Results are displayed in Figs. 4 and 5. Cases II and IV yield a highly satisfactory fit. Results are slightly less accurate for a relatively large vol--vol parameter (Case I) or term structure (Case III). The reason for this is that the truncation error corresponding to the Taylor approximation of $\operatorname{erf}(z)$ in (4.9) increases for larger $z$ values, see Remark 4.5. This is a minor issue, as for typical FX markets $\omega(t)$ and - for relatively large expiries $\gamma(t)$ assume moderate values, which are comparable with the values in Case IV.

Table 3. Effective parameter values.

\begin{tabular}{cccc}
\hline Case & I & II & III \\
\hline$t$ & 5 & 5 & 5 \\
$\widetilde{\gamma}$ & 0.679 & 0.316 & 0.316 \\
$\widetilde{\omega}$ & 0.157 & 0.156 & 0.466 \\
\hline
\end{tabular}

\begin{tabular}{ccccc}
\hline \multicolumn{5}{c}{ Case IV } \\
\hline$t$ & 1 & 2 & 3 & 5 \\
$\widetilde{\gamma}$ & 0.937 & 0.803 & 0.707 & 0.575 \\
$\widetilde{\omega}$ & 0.110 & 0.123 & 0.132 & 0.146 \\
\hline
\end{tabular}




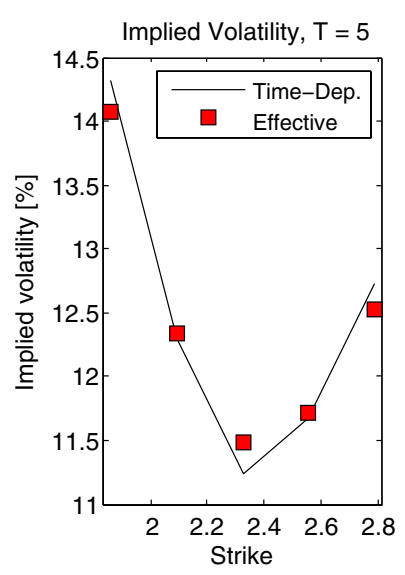

(a)

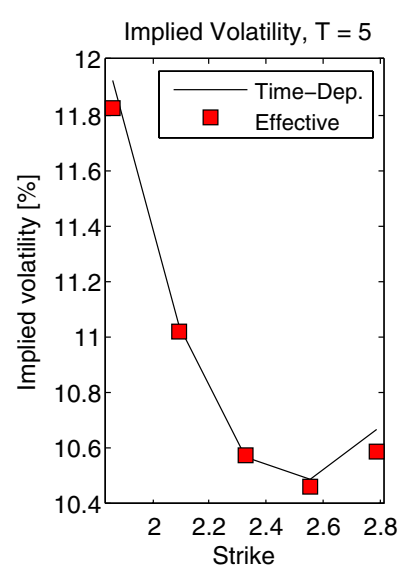

(b)

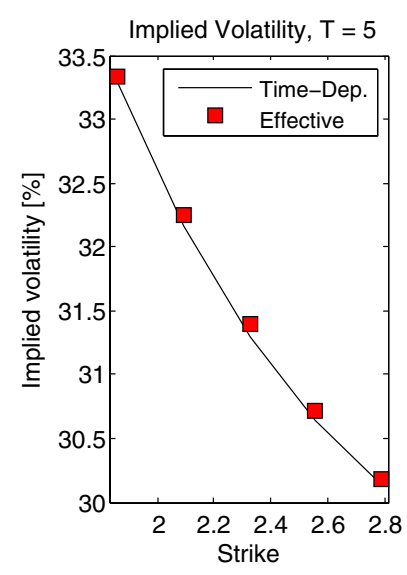

(c)

Fig. 4. Stand-alone performance of the effective term structure parameter for Cases I-III, $T=5$ $(\gamma(\cdot)$ is constant over time).

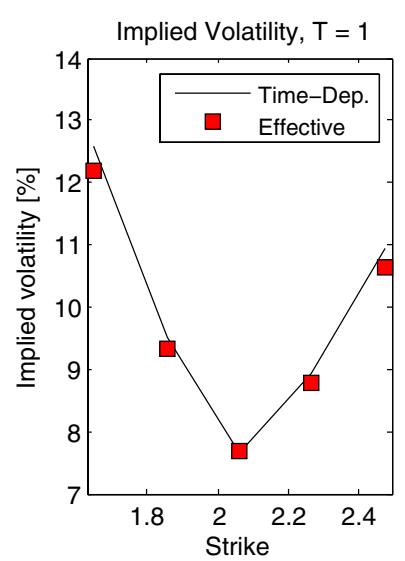

(a)

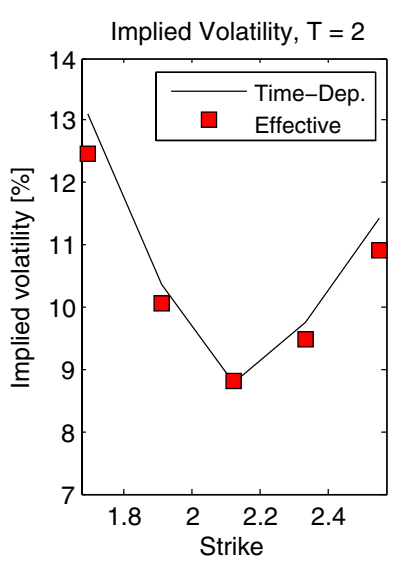

(b)

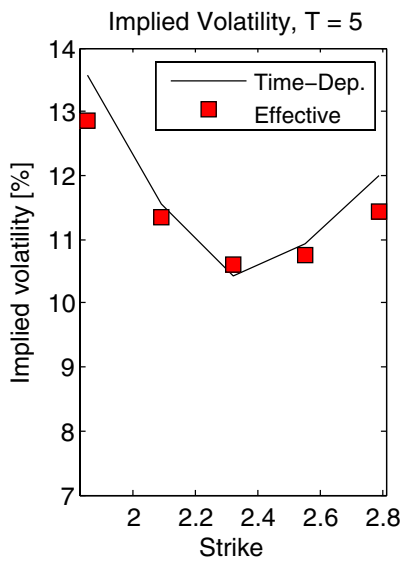

(c)

Fig. 5. Combined performance of the effective vol-vol and term structure parameters for Case IV, $T=1$ (a), $T=2(\mathrm{~b})$ and $T=5$ (c).

\subsection{Effective correlation}

At this point, we have determined the effective vol-vol and term structure parameters. As the numerical experiments in Sec. 4.2.1 show, the combined use of these effective parameters yields an accurate fit in both curvature and level. In the experiments, we assumed zero correlation and set $\beta=0.5$. By assuming these parameters to be constant, a time-dependent skew effect cannot be accounted for. To resolve this, we derive effective correlation.

We obtain the effective correlation by considering the vanna skew of the SABR model. Based on an approximation of their main pricing formula ("Hagan's 
formula"), Hagan et al. (2002) define the vanna skew (corresponding to the effective model (3.1) and (3.2) as $^{\mathrm{o}}$ :

$$
\widetilde{\nu}\left(\widetilde{\rho}_{y, \sigma}\right):=\frac{1}{2} \widetilde{\rho}_{y, \sigma} \widetilde{\lambda} \log \left(\frac{K}{\bar{y}_{0}^{T_{i}}}\right), \quad \widetilde{\lambda}=\frac{\widetilde{\gamma}}{\widetilde{\omega}}\left(\bar{y}_{0}^{T_{i}}\right)^{1-\beta} .
$$

The vanna skew is the part of the skew which is caused by $\widetilde{\rho}_{y, \sigma}$. The other part of the skew is mainly caused by the skew parameter $\beta$ (see Remark 4.9 ). The parameter $\tilde{\lambda}$ measures the "strength" of the vol-vol parameter $\widetilde{\gamma}$ compared to the local volatility, $\widetilde{\omega} /\left(\bar{y}_{0}^{T_{i}}\right)^{1-\beta}$.

We define the effective correlation corresponding to the expiry $T_{i}$ as:

$$
\widetilde{\rho}_{y, \sigma}:=\arg \min _{\rho}\left(\widetilde{\nu}(\rho)-\frac{1}{T_{i}} \int_{0}^{T_{i}} \nu\left(\rho_{y, \sigma}(t)\right) \mathrm{d} t\right),
$$

with

$$
\nu\left(\rho_{y, \sigma}(t)\right):=\frac{1}{2} \rho_{y, \sigma}(t) \lambda(t) \log \left(\frac{K}{\bar{y}_{0}^{T_{i}}}\right), \quad \lambda(t)=\frac{\gamma(t)}{\omega(t)}\left(\bar{y}_{0}^{T_{i}}\right)^{1-\beta} .
$$

In other words, the effective correlation $\widetilde{\rho}_{y, \sigma}$ is defined as the correlation value for which the vanna skew corresponding to the effective model at $t=T_{i}$ equals the average vanna skew corresponding to the time-dependent model over $\left[0, T_{i}\right]$.

Lemma 4.6 (Effective correlation). Suppose that the effective term structure $\widetilde{\omega}$ and effective vol-vol parameter $\widetilde{\gamma}$ have been established. From definition (4.25) it follows that the effective correlation corresponding to the expiry $T_{i}$ is given by:

$$
\widetilde{\rho}_{y, \sigma}=\frac{\widetilde{\omega}}{\widetilde{\gamma} T_{i}} \int_{0}^{T_{i}} \frac{\rho_{y, \sigma}(t) \gamma(t)}{\omega(t)} \mathrm{d} t .
$$

Proof. By its definition (4.25), we obtain the effective correlation by solving

$$
\begin{aligned}
\widetilde{\nu}\left(\widetilde{\rho}_{y, \sigma}\right) & =\frac{1}{T_{i}} \int_{0}^{T_{i}} \nu\left(\rho_{y, \sigma}(t)\right) \mathrm{d} t \\
\Leftrightarrow \frac{1}{2} \widetilde{\rho}_{y, \sigma} \widetilde{\lambda} \log \left(\frac{K}{\bar{y}_{0}^{T_{i}}}\right) & =\frac{1}{2 T_{i}} \log \left(\frac{K}{\bar{y}_{0}^{T_{i}}}\right) \int_{0}^{T_{i}} \rho_{y, \sigma}(t) \lambda(t) \mathrm{d} t,
\end{aligned}
$$

with

$$
\lambda(t)=\frac{\gamma(t)}{\omega(t)}\left(\bar{y}_{0}^{T_{i}}\right)^{1-\beta}
$$

Equation (4.27) yields

$$
\tilde{\rho}_{y, \sigma}=\frac{1}{\widetilde{\lambda} T_{i}} \int_{0}^{T_{i}} \rho_{y, \sigma}(t) \lambda(t) \mathrm{d} t=\frac{\widetilde{\omega}}{\widetilde{\gamma} T_{i}} \int_{0}^{T_{i}} \frac{\rho_{y, \sigma}(t) \gamma(t)}{\omega(t)} \mathrm{d} t .
$$

The effective correlation parameter is independent of the initial forward and $\beta$.

${ }^{\circ}$ We have defined model (2.3) and (2.4) such that $\bar{y}_{0}^{T_{i}}=1$ and we can simplify the formulas. However, for the sake of completeness we include the term $\bar{y}_{0}^{T_{i}}$. 
Remark 4.9 (Effective skew parameter). Hagan et al. (2002) determine $-\frac{1}{2}(1-\beta) \log \left(K / \bar{y}_{0}^{T_{i}}\right)$ as being the skew implied by the skew parameter. In a similar fashion as for the effective correlation, we may derive an effective skew parameter. We do not include the derivations though, as we do not assume a time-dependent skew parameter.

\subsubsection{Numerical experiment}

In this section, we test the combined performance of the effective vol-vol parameter $\widetilde{\gamma}$, effective term structure $\widetilde{\omega}$ and effective correlation $\widetilde{\rho}_{y, \sigma}$ (the Monte Carlo simulation consists of $5 \cdot 10^{5}$ paths and 200 time-steps per year).

Let $y_{0}=2, \beta=0.5, r_{d}=0.05$ and $r_{f}=0.02$. Time-dependent and effective parameter values are provided by Table 4 . Results are highly satisfactory, see Fig. 6 .

\section{Calibration and Pricing}

In this section, we calibrate the time-dependent FX-SABR model to market data. We compare calibration results for this model and the constant-parameter FXSABR and local volatility models. We perform three calibration experiments.

Table 4. Time-dependent and effective parameter values.

\begin{tabular}{|c|c|c|c|c|c|c|c|c|c|c|c|}
\hline$t$ & {$\left[0, \frac{1}{2}\right)$} & {$\left[\frac{1}{2}, 1\right)$} & {$[1,2)$} & {$[2,3)$} & {$[3,5]$} & $t$ & $\frac{1}{2}$ & 1 & 2 & 3 & 5 \\
\hline$\gamma(t)$ & 1 & 0.8 & 0.5 & 0.3 & 0.2 & $\widetilde{\gamma}$ & 1.000 & 0.937 & 0.803 & 0.707 & 0.575 \\
\hline$\omega(t)$ & 0.1 & 0.12 & 0.14 & 0.16 & 0.18 & $\tilde{\widetilde{\omega}}$ & 0.100 & 0.110 & 0.123 & 0.132 & 0.146 \\
\hline$\rho_{y, \sigma}(t)$ & -0.9 & -0.8 & -0.7 & -0.6 & -0.5 & $\tilde{\rho}_{y, \sigma}$ & -0.900 & -0.840 & -0.739 & -0.673 & -0.606 \\
\hline
\end{tabular}

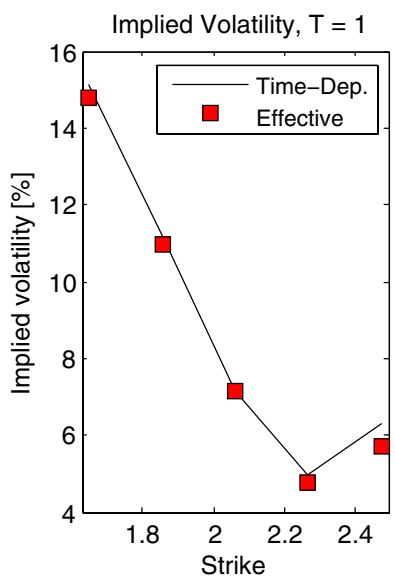

(a)

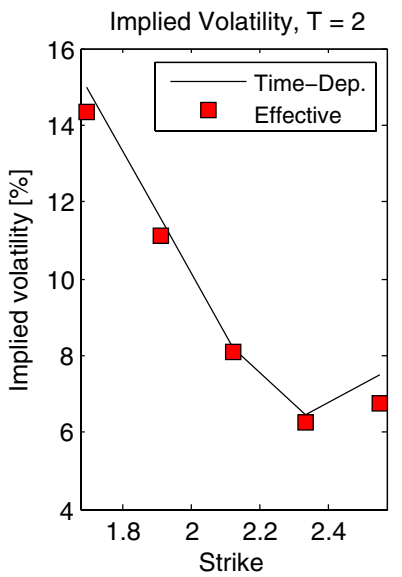

(b)

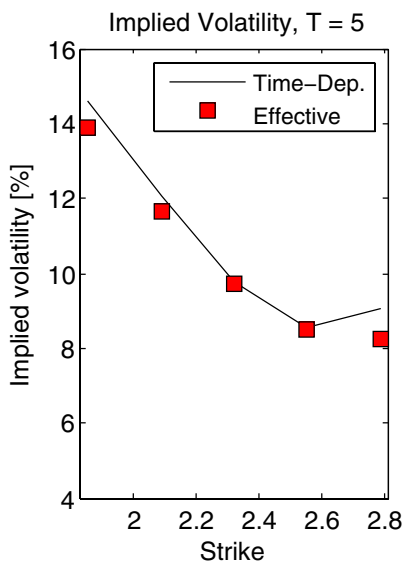

(c)

Fig. 6. Combined performance of the effective vol-vol parameter, effective term structure and effective correlation for $T=1$ (a), $T=2$ (b) and $T=5$ (c). 
In each experiment, we calibrate the time-dependent model to two expiries. Subsequently, in Sec. 5.3, we price barrier options with corresponding times to maturity.

We consider USD/AUD FX market prices quoted on 12 June, 2013 from a market data vendor. Domestic currency is USD, foreign currency is AUD. Initial spot is $y_{0}=0.9548$. Implied volatilities are quoted for five different strikes. The third strike corresponds to the ATM level. Data is provided for expiries between one day and five years. The ATM term structure exhibits a downward trend up to one year. For longer expiries, the ATM level moves in opposite direction. Also, an increasingly pronounced skew can be observed over time, whereas the amount of curvature slightly declines. Further, the market data consists of mid-prices and bid-ask spreads of up-out put options with expiries of three months, one year and two years.

Remark 5.1 (Deterministic interest rates). In Sec. 2, we introduce the timedependent FX-SABR model with deterministic interest rates. The deterministic interest rates assumption merely serves the purpose of transparency and readability. Assuming stochastic interest rates would complicate notation. The mappings of the effective parameters are not affected by the deterministic interest rates assumption. As our numerical experiments in this section confirm, the effective parameters facilitate accurate calibration to the FX market, also for longer times to maturity when stochastic interest rate effects become more important.

\subsection{Calibration procedure}

The (implicit) mappings derived for effective parameters imply the following functional dependencies:

$$
\begin{aligned}
& \widetilde{\gamma}^{\text {mod }}=f_{1}(\gamma(t), \omega(t)), \\
& \widetilde{\omega}^{\text {mod }}=f_{2}\left(\gamma(t), \omega(t), \widetilde{\gamma}^{\text {mod }}\right), \\
& \widetilde{\rho}_{y, \sigma}^{\text {mod }}=f_{3}\left(\gamma(t), \omega(t), \rho_{y, \sigma}(t), \widetilde{\gamma}^{\text {mod }}, \widetilde{\omega}^{\text {mod }}\right),
\end{aligned}
$$

where $\gamma(t), \omega(t)$ and $\rho_{y, \sigma}(t)$ are time-dependent parameters in the time-dependent model (2.1) and (2.2) and $\widetilde{\gamma}^{\bmod }, \widetilde{\omega}^{\bmod }$ and $\widetilde{\rho}_{y, \sigma}^{\bmod }$ are their "effective equivalents". Similar as in the experiments in Secs. 4.1.1, 4.2.1 and 4.3.1, we assume that the time-dependent parameters are piecewise-constant.

The calibration consists of four stages. First, we calibrate the effective SABR model (3.1) and (3.12) using Hagan's formulas. ${ }^{\mathrm{p}}$ For each expiry, we obtain a set of market effective parameters $\left\{\widetilde{\gamma}^{\text {mar }}, \widetilde{\omega}^{\text {mar }}, \widetilde{\rho}_{y, \sigma}^{\text {mar }}\right\}$ - see also Sec. 3. Second, we

\footnotetext{
PIt is well known that Hagan's formulas are biased for extreme strikes and large times to maturity, see e.g. (Islah 2009, Oblój 2008, Paulot 2009, Takahashi et al. 2007). In Antonov et al. (2013) it is pointed out that for maturities larger than 10 years, the error in implied volatility can be $1 \%$ or more, even for ATM values. However, in this article no bias as a result of Hagan's formulas is introduced. We have confirmed this for the effective model by assuming zero correlation and applying the analytical pricing method of Antonov et al. (2013). We obtain the same ATM level, which implies that our results are free of bias due to Hagan's formula or the Monte Carlo simulation (correlation does not affect smile level, see Sec. 3).
} 
calibrate the time-dependent SABR model (2.1) and (2.2). As $\gamma(t)$ and $\rho_{y, \sigma}(t)$ both have a curvature (and skew) effect, we calibrate these parameters simultaneously on the base of implied volatilities. As at this point $\omega(t)$ is not established yet, we apply the approximations

$$
\begin{gathered}
f_{1}(\gamma(t), \omega(t)) \approx f_{1}\left(\gamma(t), \widetilde{\omega}^{\mathrm{mar}}\right), \\
f_{3}\left(\gamma(t), \omega(t), \rho_{y, \sigma}(t), \widetilde{\gamma}^{\mathrm{mod}}, \widetilde{\omega}^{\mathrm{mod}}\right) \approx f_{3}\left(\gamma(t), \rho_{y, \sigma}(t), \widetilde{\gamma}^{\mathrm{mod}}, \widetilde{\omega}^{\mathrm{mar}}\right) .
\end{gathered}
$$

Successively, we calibrate $\omega(t)$ in order to obtain a level fit. Third, to compensate for the approximation (5.2) in the effective correlation mapping $f_{3}(\cdot)$, we perform another calibration iteration for $\rho_{y, \sigma}(t)$ separately, given the values for $\gamma(t)$ and $\omega(t)$ obtained in the second calibration stage. We observe that an additional calibration iteration for $\gamma(t)$ (to compensate for the $f_{1}(\cdot)$ mapping approximation $(5.1)$ ) does not yield a significant improvement in results. In the fourth stage, we add the local volatility component $\sigma_{\mathrm{SLV}}(\cdot)$ specified in Sec. 2.2 on top of the time-dependent $\mathrm{SABR}$ model to compensate for calibration inaccuracies. We summarize the calibration routine in Algorithm 1.

Algorithm 1. Calibration procedure.

\section{Calibration routine:}

1 Calibrate the effective SABR model (3.1) and (3.2). This yields market effective parameters $\left\{\widetilde{\gamma}_{i}^{\operatorname{mar}}, \widetilde{\omega}_{i}^{\operatorname{mar}}, \widetilde{\rho}_{y, \sigma, i}^{\operatorname{mar}}\right\}, i=1, \ldots, N$.

2 Calibrate $\rho_{y, \sigma}(t)$ and $\gamma(t)$ simultaneously. For this the mapping approximations (5.1) and (5.2) are required. Successively, calibrate $\omega(t)$ separately in order to obtain a level fit.

3 Calibrate $\rho_{y, \sigma}(t)$ separately, given the values for $\omega(t)$ and $\gamma(t)$ just obtained. Now the original mapping on the left-hand side of (5.2) can be applied.

4 Add the local volatility component $\sigma_{\mathrm{SLV}}(\cdot)$ to compensate for calibration inaccuracies.

\subsection{Calibration results}

In Tables 5-7, the calibration errors are given, defined as $\sigma_{i, \bmod }-\sigma_{i, \operatorname{mar}}$, where $\sigma_{i, \bmod }$ and $\sigma_{i, \text { mar }}$ are the corresponding model and market implied volatilities in percentages, respectively ( $i$ denotes the strike). The total absolute error $\varepsilon_{\text {tot }}:=$ $\sum_{i=1}^{5}\left|\sigma_{i, \bmod }-\sigma_{i, \operatorname{mar}}\right|$ is also provided. The results (also for the constant-parameter SABR model) are obtained by Monte Carlo simulation runs consisting of $5 \cdot 10^{5}$ paths and 1000 time-steps per year for Experiment I and 200 time-steps per year for Experiments II and III.

For the local volatility model and the time-dependent model with the local volatility component, the calibration results are most accurate. Comparing the 
Table 5. Calibration errors in Experiment I (in \%). $\varepsilon_{\text {tot }}:=$ $\sum_{i=1}^{5}\left|\sigma_{i, \bmod }-\sigma_{i, \operatorname{mar}}\right|$, where $i$ indicates strike.

\begin{tabular}{cccccc}
\hline Expiry & Strike & Time-dep.+LV & Time-dep. & \multicolumn{1}{c}{ LV } & Constant \\
\hline $2 \mathrm{M}$ & 0.87 & 0.031 & 0.276 & 0.109 & -0.802 \\
& 0.91 & -0.037 & -0.011 & -0.011 & -0.655 \\
& 0.95 & 0.015 & 0.042 & 0.035 & -0.431 \\
& 0.98 & 0.016 & 0.289 & 0.040 & -0.347 \\
& 1.01 & -0.026 & 0.616 & 0.020 & -0.404 \\
& $\varepsilon_{\text {tot }}$ & $\mathbf{0 . 1 2 5}$ & $\mathbf{1 . 2 3 4}$ & $\mathbf{0 . 2 1 5}$ & $\mathbf{2 . 6 3 8}$ \\
$3 \mathrm{M}$ & 0.85 & -0.050 & 0.346 & 0.110 & -0.195 \\
& 0.91 & -0.100 & -0.033 & -0.017 & -0.164 \\
& 0.95 & -0.025 & 0.012 & 0.035 & 0.004 \\
& 0.99 & -0.015 & 0.361 & 0.044 & 0.095 \\
& 1.02 & -0.081 & 0.821 & 0.022 & 0.089 \\
& $\varepsilon_{\text {tot }}$ & $\mathbf{0 . 2 7 2}$ & $\mathbf{1 . 5 7 2}$ & $\mathbf{0 . 2 2 8}$ & $\mathbf{0 . 5 4 8}$ \\
\hline
\end{tabular}

Table 6. Calibration errors in Experiment II (in \%). $\varepsilon_{\text {tot }}:=$ $\sum_{i=1}^{5}\left|\sigma_{i, \bmod }-\sigma_{i, \operatorname{mar}}\right|$, where $i$ indicates strike.

\begin{tabular}{cccccc}
\hline Expiry & Strike & Time-dep.+LV & Time-dep. & \multicolumn{1}{c}{ LV } & Constant \\
\hline $6 \mathrm{M}$ & 0.81 & -0.029 & -0.261 & 0.166 & -1.303 \\
& 0.89 & -0.084 & -0.208 & -0.003 & -0.959 \\
& 0.94 & -0.013 & 0.047 & 0.037 & -0.476 \\
& 1.00 & 0.039 & 0.301 & 0.091 & -0.213 \\
& 1.04 & -0.080 & 0.314 & -0.002 & -0.424 \\
& $\varepsilon_{\text {tot }}$ & $\mathbf{0 . 2 4 6}$ & $\mathbf{1 . 1 3 1}$ & $\mathbf{0 . 2 9 8}$ & $\mathbf{3 . 3 7 4}$ \\
$1 \mathrm{Y}$ & 0.76 & -0.088 & 0.222 & 0.160 & -0.443 \\
& 0.86 & -0.167 & -0.105 & -0.063 & -0.325 \\
& 0.93 & -0.046 & -0.038 & 0.028 & -0.032 \\
& 1.00 & 0.035 & 0.246 & 0.113 & 0.171 \\
& 1.07 & -0.127 & 0.409 & -0.032 & 0.016 \\
& $\varepsilon_{\text {tot }}$ & $\mathbf{0 . 4 6 3}$ & $\mathbf{1 . 0 1 9}$ & $\mathbf{0 . 3 9 6}$ & $\mathbf{0 . 9 8 6}$ \\
\hline
\end{tabular}

Table 7. Calibration errors in Experiment III (in \%). $\varepsilon_{\text {tot }}:=$ $\sum_{i=1}^{5}\left|\sigma_{i, \bmod }-\sigma_{i, \operatorname{mar}}\right|$, where $i$ indicates strike.

\begin{tabular}{ccccrr}
\hline Expiry & Strike & Time-dep.+LV & Time-dep. & \multicolumn{1}{c}{ LV } & Constant \\
\hline $1 \mathrm{Y}$ & 0.76 & 0.002 & -0.769 & 0.153 & -1.638 \\
& 0.86 & -0.098 & -0.406 & -0.066 & -0.674 \\
& 0.93 & 0.020 & 0.059 & 0.025 & 0.114 \\
& 1.00 & 0.102 & 0.291 & 0.102 & 0.432 \\
& 1.07 & -0.062 & -0.003 & -0.047 & -0.039 \\
& $\varepsilon_{\text {tot }}$ & $\mathbf{0 . 2 8 5}$ & $\mathbf{1 . 5 2 9}$ & $\mathbf{0 . 3 9 3}$ & $\mathbf{2 . 8 9 8}$ \\
& & & & & \\
& 0.69 & 0.014 & 0.314 & 0.155 & -0.410 \\
& 0.82 & -0.100 & -0.060 & -0.068 & -0.326 \\
& 0.91 & 0.029 & -0.024 & 0.038 & -0.047 \\
& 1.01 & 0.144 & 0.230 & 0.140 & 0.173 \\
& 1.11 & -0.030 & 0.362 & -0.038 & -0.007 \\
& $\varepsilon_{\text {tot }}$ & $\mathbf{0 . 3 1 7}$ & $\mathbf{0 . 9 9 0}$ & $\mathbf{0 . 4 3 9}$ & $\mathbf{0 . 9 6 5}$ \\
\hline
\end{tabular}


calibration errors of the time-dependent model with and without local volatility component, we observe that the local volatility component reduces the calibration error. By its construction, it naturally "bridges" the mismatch between the market and (time-dependent FX-SABR) model prices (see e.g. Van den Stoep et al. (2014). Further, except for Experiment I, the constant-parameter and timedependent SABR models, overall yield similar calibration accuracy for the second expiry. For the constant-parameter SABR model, the calibration error is substantial for the intermediate expiry, as we calibrate this model to the last expiry. For illustration purposes, the implied volatility smiles corresponding to Experiment III are displayed in Fig. 7. The calibration of the time-dependent model is accurate. Inclusion of the non-parametric local volatility component yields a highly satisfactory fit to the market. Similar figures were obtained for Experiments I and II.

Calibrated parameter values are provided by Table 8 . The time-dependent volvol parameter decreases over time, which implies that curvature in the model implied volatility surface declines. This effect was also observed in the market data. Further, the term structure parameter decreases in Experiments I and II and increases in Experiment III, which is in line with the observation in the market of a decreasing term structure up to 1 year and an increase for longer expiries. Further, the correlation becomes more negative, which implies a more pronounced skew effect implied by the model. The market surface exhibits the same feature.

Remark 5.2 (Multiple expiries). We only show and discuss the barrier pricing results for the model that we calibrated to two expiries in Experiments I, II and III. The reason for this is the following. For this particular dataset, the improvement in the pricing of barrier options (see the follow-up section) turns out to be marginal

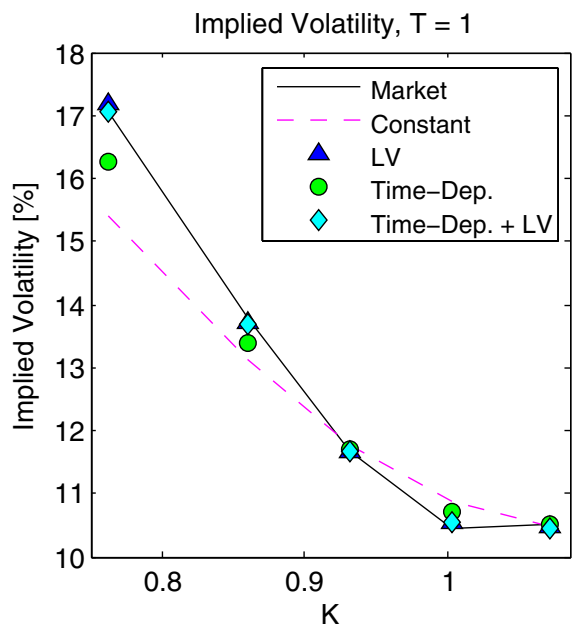

(a)

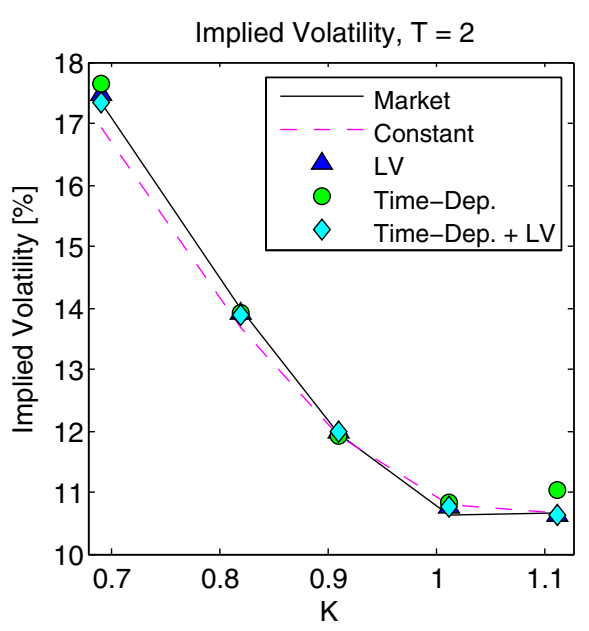

(b)

Fig. 7. Black-Scholes implied volatility smiles for Experiment III: one year (a) and two years (b). 
Table 8. Calibrated piecewise-constant parameter values.

\begin{tabular}{ccccccc}
\hline Exper. & $\gamma_{1}$ & $\gamma_{2}$ & $\omega_{1}$ & $\omega_{2}$ & $\rho_{1}$ & $\rho_{2}$ \\
\hline I & 1.597 & 0.468 & 0.117 & 0.107 & -0.389 & -0.996 \\
II & 1.008 & 0.468 & 0.110 & 0.100 & -0.440 & -0.611 \\
III & 0.695 & 0.203 & 0.106 & 0.111 & -0.456 & -0.944 \\
\hline
\end{tabular}

when we calibrate the model to more than two expiries. This is a property of this specific dataset and not a model property: for this dataset we do not need to calibrate to more than 2 expiries, although the model - by means of the effective parameters derived - can be calibrated accurately to multiple expiries if necessary. In Appendix B we calibrate to 4 expiries, namely 1, 2, 3 and 4 years.

As an indication regarding CPU times, we calibrate piecewise-constant $\omega(t)$, $\gamma(t)$ and $\rho_{y, \sigma}(t)$ for Experiment III in approximately $14 \mathrm{~s}$ (in Matlab on an i5-2400 $\mathrm{CPU}$ at $3.10 \mathrm{GHz}, 3101 \mathrm{MHz}, 4$ Cores, 4 Logical Processors).

\subsection{Pricing barrier options}

In this section, we price barrier options by the local volatility model, the constantparameter SABR model and the time-dependent SABR model with and without local volatility component. In contrast to European-type options, the prices of barrier options do not only depend on the distribution of the underlying at the time to maturity, but also on the dynamics of the implied volatility smile through time (Tataru \& Fisher 2010). The prices are determined by the underlying's future transition densities, which are reflected by the forward implied volatility smiles a particular model produces (Baker et al. 2004). The dynamics of the implied volatility smile with respect to the underlying are also relevant, especially for hedging purposes.

In this section, we price up-out put options with different strikes and barriers. The value of an up-out put option is given as follows. Define $B$ as the barrier level and assume for the initial spot FX rate $y_{0}<B$. The discounted value of an up-out put option with strike $K$ and expiry $T$ at at an arbitrary time $t \in[0, T]$ reads

$$
P_{\mathrm{UO}}(t, T, K):=\mathbb{E}^{\mathbb{Q}}\left[\frac{M_{d}(t)}{M_{d}(T)}(K-y(T))^{+} \mathbb{1}\left(\max _{t \in[0, T]} y(t)<B\right) \mid \mathcal{F}(t)\right],
$$

where $M_{d}(\cdot)$ is the domestic moneyness account determined by $\mathrm{d} M_{d}(t)=$ $r_{d}(t) M_{d}(t) \mathrm{d} t$, with $r_{d}(\cdot)$ denoting the domestic interest rate. In the numerical experiments, we assume $K<y_{0}<B$, so each option starts out-of-the-money. Pricing results are provided in Table 9 .

In each experiment, the local volatility model implies significantly higher prices compared to the other stochastic volatility models: 6 of 9 prices are higher than the ask price observed in the market. Each of the models with stochastic volatility only yields one price that is not within the bid-ask spread. Also, the errors corresponding to the stochastic volatility models are significantly smaller. This suggests that the 
Table 9. Model prices up-out put options multiplied by a factor $10^{4}$. Results in brackets are not within bid-ask spread. The error is defined as $\varepsilon_{\text {tot }}:=\sum_{i=1}^{9}\left|B_{i, \bmod }-B_{i, \operatorname{mar}}\right|$, where $i$ indicates the particular barrier option and $B_{i, \bmod }$ and $B_{i, \text { mar }}$ are the corresponding model and mid-market prices, respectively.

\begin{tabular}{|c|c|c|c|c|c|c|c|}
\hline Exper. & Expiry & Barrier & Strike & LV & Constant & Time-dep. & Time-dep.+LV \\
\hline \multirow[t]{3}{*}{ I } & $3 \mathrm{M}$ & 1 & 0.9 & 97.53 & 92.76 & 91.12 & 90.39 \\
\hline & $3 \mathrm{M}$ & 1 & 0.85 & 37.41 & 34.51 & 36.50 & 33.08 \\
\hline & $3 \mathrm{M}$ & 0.97 & 0.9 & $(70.12)$ & 62.91 & 60.35 & 59.54 \\
\hline \multirow[t]{3}{*}{ II } & $1 \mathrm{Y}$ & 1 & 0.8 & (114.17) & 92.71 & 95.40 & 93.08 \\
\hline & $1 \mathrm{Y}$ & 1 & 0.85 & (178.39) & $(153.26)$ & (152.67) & (152.11) \\
\hline & $1 \mathrm{Y}$ & 0.97 & 0.8 & $(72.05)$ & 54.19 & 55.48 & 54.55 \\
\hline \multirow[t]{4}{*}{ III } & $2 \mathrm{Y}$ & 1 & 0.75 & (165.25) & 129.96 & 135.67 & 134.24 \\
\hline & $2 \mathrm{Y}$ & 1.05 & 0.7 & 140.48 & 117.44 & 127.46 & 121.33 \\
\hline & $2 \mathrm{Y}$ & 0.97 & 0.75 & $(91.82)$ & 69.14 & 72.16 & 72.08 \\
\hline & & & $\varepsilon_{\text {tot }}$ & 146.00 & 51.43 & 38.97 & 46.53 \\
\hline
\end{tabular}

market consensus seems to price barrier options with a "SABR-like" stochastic volatility model, instead of the local volatility model. A plausible reason for this is the fact that the latter typically does not accurately "capture" the forward implied volatility smile (Engelmann et al. 2011, Gatheral 2011). As we mentioned at the beginning of this section, e.g. in Tataru \& Fisher (2010) the relevance of the smile dynamics for the pricing of path-dependent derivatives is pointed out. Besides for this, the predicted implied volatility smile moves with respect to the underlying are also relevant, especially for the hedging of complex products (Hagan et al. 2002, Johnson \& Lee 2003). ${ }^{\mathrm{q}}$

To price the up-out put option correctly, the model should not only imply the correct smile dynamics. It should also price plain vanilla options accurately across a grid of multiple expiries, as these provide information about market behavior over time. In Tables 5-7, we observed that the constant-parameter SABR model is only well-calibrated to the last expiry. This is trivial, as the calibrated parameter values only correspond to this maturity. By assuming time-dependent parameters, the calibration error at an intermediate expiry is reduced significantly: The plain vanillas are priced more accurately across multiple expiries. We observe that the time-dependent model yields prices which are closer to the reference compared to the constant-parameter SABR model.

\footnotetext{
qBarrier options can be prices and hedged in a dynamic or (quasi-)static way. Dynamic hedging models price an exotic option on the base of the costs of dynamically hedging the product with a portfolio of the underlying asset and European-type options (in an analogous way, the BlackScholes model prices vanilla European options based on the costs of a time-dependent hedge with the underlying asset). In this approach the hedging risks and costs may be substantial, as pointed out by e.g. Derman et al. (1995). As an alternative, they adopt a static hedging model, which values the barrier option based on the cost of a replication strategy which requires an unchanging hedge portfolio consisting of European-type options.
} 
By incorporating the non-parametric local volatility component, the timedependent model yields less accurate up-out put option prices. This may be due to the fact that the local volatility model predicts inaccurate smile dynamics. However, the calibration error reduces significantly (see Tables 5-7). We therefore tend to prefer the model with local volatility component, as it may reduce the additional costs of hedging the barrier option due to a better calibration performance.

Last, the results indicate that for each model and expiry, the up-out put option prices are consistent across different barriers and strikes. More specifically, each model yields a higher up-out put option price for a higher barrier and/or higher strike. This is intuitive, as a higher barrier implies a smaller probability that the barrier is reached and that the option becomes worthless. Also, a higher strike means that the underlying can be sold for a larger amount of money.

\section{Conclusion}

In this paper, we have presented a framework for accurate and efficient calibration of the time-dependent SABR model in an FX context. By considering the effects of the SABR parameters on the shape of the implied volatility smile, we have derived "effective equivalents" of the time-dependent vol-vol, term structure and correlation parameters. Numerical experiments show that both the separate and combined performances of the effective parameters are accurate, which results into highly satisfactory calibration results. A non-parametric local volatility component can compensate for the calibration inaccuracies. In our barrier option pricing experiments, the time-dependent FX-SABR model outperforms the traditional local volatility and constant-parameter SABR models. Our results seem to indicate that there is a market consensus of pricing barrier options by a "SABR-like" stochastic volatility model. Plausible reasons are the facts that the local volatility model typically does not accurately "capture" the forward implied volatility smile and the implied volatility smile moves with respect to the underlying, which are relevant features for the accurate pricing and hedging of path-dependent derivatives.

\section{Appendix A: Characteristic Function Recovery}

Define

$$
R_{j}:=\log \left(\frac{\omega_{1}^{2}\left(t_{j}\right) \sigma^{2}\left(t_{j}\right)}{\omega_{1}^{2}\left(t_{j-1}\right) \sigma^{2}\left(t_{j-1}\right)}\right), \quad j=1, \ldots, M
$$

and stochastic process $Y_{j}$, which is given by

$$
\begin{aligned}
Y_{j}:= & \log \left(\frac{\omega_{1}^{2}\left(t_{M-j+1}\right) \sigma^{2}\left(t_{M-j+1}\right)}{\omega_{1}^{2}\left(t_{M-j}\right) \sigma^{2}\left(t_{M-j}\right)}+\frac{\omega_{1}^{2}\left(t_{M-j+2}\right) \sigma^{2}\left(t_{M-j+2}\right)}{\omega_{1}^{2}\left(t_{M-j}\right) \sigma^{2}\left(t_{M-j}\right)}\right. \\
& \left.+\cdots+\frac{\omega_{1}^{2}\left(t_{M}\right) \sigma^{2}\left(t_{M}\right)}{\omega_{1}^{2}\left(t_{M-j}\right) \sigma^{2}\left(t_{M-j}\right)}\right) .
\end{aligned}
$$


It is easy to see that

$$
Y_{1}=\log \left(\frac{\omega_{1}^{2}\left(t_{M}\right) \sigma^{2}\left(t_{M}\right)}{\omega_{1}^{2}\left(t_{M-1}\right) \sigma^{2}\left(t_{M-1}\right)}\right)=R_{M}
$$

and

$$
Y_{M}=\log \left(\sum_{j=1}^{M} \frac{\omega_{1}^{2}\left(t_{j}\right) \sigma^{2}\left(t_{j}\right)}{\omega_{1}^{2}(0) \sigma_{0}^{2}}\right)=\log \left(\sum_{j=1}^{M} \frac{\omega_{1}^{2}\left(t_{j}\right) \sigma^{2}\left(t_{j}\right)}{\omega_{1}^{2}(0)}\right)
$$

as $\sigma_{0}=1$. To recover the approximated characteristic function $\widehat{\phi}_{Y_{M}}(\cdot)$ corresponding to $Y_{M}$, we need to know the distribution of $R_{j}, j=1, \ldots, M$ in (A.1).

\section{A.1. Distribution of $\boldsymbol{R}_{j}$}

We determine the distribution of $R_{j}, j=1, \ldots, M$ defined in (A.1) by first deriving the dynamics of $Z(t):=\omega_{1}^{2}(t) \sigma^{2}(t)$. As the $\sigma^{2}(t)$-dynamics are given by

$$
\mathrm{d} \sigma^{2}(t)=\sigma^{2}(t)\left(\gamma^{2}(t) \mathrm{d} t+2 \gamma(t) \mathrm{d} W_{\sigma}(t)\right),
$$

we obtain

$$
\begin{aligned}
\mathrm{d} Z(t) & =\left[\mathrm{d} \omega_{1}^{2}(t)\right] \sigma^{2}(t)+\omega_{1}^{2}(t) \mathrm{d} \sigma^{2}(t)+\mathrm{d} \omega_{1}^{2}(t) \mathrm{d} \sigma^{2}(t) \\
& =\left(\gamma^{2}(t)+\frac{\mathrm{d} \omega_{1}^{2}(t)}{\mathrm{d} t} \frac{1}{\omega_{1}^{2}(t)}\right) Z(t) \mathrm{d} t+2 \gamma(t) Z(t) \mathrm{d} W_{\sigma}(t) .
\end{aligned}
$$

The solution then leads

$$
Z(t)=Z_{0} \exp \left(\int_{0}^{t}-\gamma^{2}(s) \mathrm{d} s+2 \int_{0}^{t} \gamma(s) \mathrm{d} W_{\sigma}(s)\right) \exp \left(\int_{0}^{t} \frac{\mathrm{d} \omega_{1}^{2}(s)}{\mathrm{d} s} \frac{1}{\omega_{1}^{2}(s)} \mathrm{d} s\right) .
$$

The integral in the second exponential term is simplified to:

$$
\int_{0}^{t} \frac{\mathrm{d} \omega_{1}^{2}(s)}{\mathrm{d} s} \frac{1}{\omega_{1}^{2}(s)} \mathrm{d} s=\int_{0}^{t} \frac{\mathrm{d} \omega_{1}^{2}(s)}{\omega_{1}^{2}(s)}=\left[\log \left(\omega_{1}^{2}(s)\right)\right]_{s=0}^{s=t}=\log \left(\frac{\omega_{1}^{2}(t)}{\omega_{1}^{2}(0)}\right)
$$

and the solution becomes ${ }^{\mathrm{r}}$ :

$$
\begin{aligned}
Z(t) & =Z_{0} \exp \left(-\int_{0}^{t} \gamma^{2}(s) \mathrm{d} s+2 \int_{0}^{t} \gamma(s) \mathrm{d} W_{\sigma}(s)\right) \frac{\omega_{1}^{2}(t)}{\omega_{1}^{2}(0)} \\
& =\omega_{1}^{2}(t) \exp \left(-\int_{0}^{t} \gamma^{2}(s) \mathrm{d} s+2 \int_{0}^{t} \gamma(s) \mathrm{d} W_{\sigma}(s)\right) .
\end{aligned}
$$

Thus, we have

$$
\begin{aligned}
R_{j} & =\log \left[\frac{\omega_{1}^{2}\left(t_{j}\right) \exp \left(-\int_{0}^{t_{j}} \gamma^{2}(s) \mathrm{d} s+2 \int_{0}^{t_{j}} \gamma(s) \mathrm{d} W_{\sigma}(s)\right)}{\omega_{1}^{2}\left(t_{j-1}\right) \exp \left(-\int_{0}^{t_{j-1}} \gamma^{2}(s) \mathrm{d} s+2 \int_{0}^{t_{j-1}} \gamma(s) \mathrm{d} W_{\sigma}(s)\right)}\right] \\
& =\log \left(\frac{\omega_{1}^{2}\left(t_{j}\right)}{\omega_{1}^{2}\left(t_{j-1}\right)}\right)-\int_{t_{j-1}}^{t_{j}} \gamma^{2}(s) \mathrm{d} s+2 \int_{t_{j-1}}^{t_{j}} \gamma(s) \mathrm{d} W_{\sigma}(s) .
\end{aligned}
$$

${ }^{\mathrm{r}}$ Equation (A.4) makes sense, as $Z(t) / \omega_{1}^{2}(t)=\exp \left(\int_{0}^{t}-\gamma^{2}(s) \mathrm{d} s+2 \int_{0}^{t} \gamma(s) \mathrm{d} W_{\sigma}(s)\right)$ or equivalently $\sigma^{2}(t)=\exp \left(-\int_{0}^{t} \gamma^{2}(s) \mathrm{d} s+2 \int_{0}^{t} \gamma(s) \mathrm{d} W_{\sigma}(s)\right)$. 
So $R_{j}$ is distributed as follows:

$$
\begin{gathered}
R_{j} \sim N\left(\mu_{R, j}, \sigma_{R, j}^{2}\right), \quad \mu_{R, j}=\log \left(\frac{\omega_{1}^{2}\left(t_{j}\right)}{\omega_{1}^{2}\left(t_{j-1}\right)}\right)-\int_{t_{j-1}}^{t_{j}} \gamma^{2}(s) \mathrm{d} s, \\
\sigma_{R, j}^{2}=4 \int_{t_{j-1}}^{t_{j}} \gamma^{2}(s) \mathrm{d} s .
\end{gathered}
$$

Hence, the characteristic function of $R_{j}$ is specified by

$$
\phi_{R_{j}}(u)=\mathrm{e}^{i u \mu_{R, j}-\frac{1}{2} u^{2} \sigma_{R, j}^{2}},
$$

with $\mu_{R, j}$ and $\sigma_{R, j}^{2}$ given above.

\section{A.2. Recovery procedure}

Given the characteristic function (A.5), we recover the characteristic function of $Y_{M}$ as follows. Let $k=0,1, \ldots, N-1$ and $l=0,1, \ldots, N-1$ and define $u_{k}:=\frac{k \pi}{b-a}$ and $u_{l}=\frac{l \pi}{b-a}$. We follow a recursion procedure, which starts at the end of the time-interval. By the definition of $Y_{j}$ in (A.2) and $R_{j}$ in (A.1), we have

$$
Y_{1}=\log \left(\frac{\omega_{1}^{2}\left(t_{M}\right) \sigma^{2}\left(t_{M}\right)}{\omega_{1}^{2}\left(t_{M-1}\right) \sigma^{2}\left(t_{M-1}\right)}\right)=R_{M}
$$

We now proceed according to the following recovery procedure:

(1) As $Y_{1}=R_{M}$, we have $\phi_{Y_{1}}\left(u_{k}\right)=\phi_{R_{M}}\left(u_{k}\right)$.

(2) If we go one step further, we obtain $\phi_{Y_{2}}\left(u_{k}\right)=\phi_{R_{M-1}}\left(u_{k}\right) \phi_{Z_{1}}\left(u_{k}\right) \approx$ $\phi_{R_{M-1}}\left(u_{k}\right) \widehat{\phi}_{Z_{1}}\left(u_{k}\right)=: \widehat{\phi}_{Y_{2}}\left(u_{k}\right)$, with

$$
\widehat{\phi}_{Z_{1}}\left(u_{k}\right)=\frac{2}{b-a} \sum_{l=0}^{N-1} \operatorname{Re}\left\{\phi_{Y_{1}}\left(u_{l}\right) \mathrm{e}^{-i a u_{l}}\right\} \int_{a}^{b}\left(\mathrm{e}^{x}+1\right)^{i u_{k}} \cos \left((x-a) u_{l}\right) \mathrm{d} x .
$$

(3) In the next iteration, we obtain $\widehat{\phi}_{Y_{3}}\left(u_{k}\right)=\phi_{R_{M-2}}\left(u_{k}\right) \widehat{\phi}_{Z_{2}}\left(u_{k}\right)$, with

$$
\widehat{\phi}_{Z_{2}}\left(u_{k}\right)=\frac{2}{b-a} \sum_{l=0}^{N-1} \operatorname{Re}\left\{\widehat{\phi}_{Y_{2}}\left(u_{l}\right) \mathrm{e}^{-i a u_{l}}\right\} \int_{a}^{b}\left(\mathrm{e}^{x}+1\right)^{i u_{k}} \cos \left((x-a) u_{l}\right) \mathrm{d} x .
$$

(4) We continue in this way until we have obtained $\widehat{\phi}_{Y_{M-1}}\left(u_{k}\right)$.

In the last, $M$ th step, we set $u=-\frac{1}{2} i$ and we calculate

$$
\widehat{\phi}_{Y_{M}}(u)=\phi_{R_{1}}(u) \widehat{\phi}_{Z_{M-1}}(u),
$$

with

$$
\widehat{\phi}_{Z_{M-1}}(u)=\frac{2}{b-a} \sum_{l=0}^{N-1} \operatorname{Re}\left\{\widehat{\phi}_{Y_{M-1}}\left(u_{l}\right) \mathrm{e}^{-i a u_{l}}\right\} \int_{a}^{b}\left(\mathrm{e}^{x}+1\right)^{i u} \cos \left((x-a) u_{l}\right) \mathrm{d} x .
$$

In the recursion, the integral $\int_{a}^{b}\left(\mathrm{e}^{x}+1\right)^{i u_{k}} \cos \left((x-a) u_{l}\right) \mathrm{d} x, k, l=0, \ldots, N-1$ has to be calculated only once (except for the $M$ th step, where $u_{k}=u$ ). One can either 
approximate it numerically by the Clenshaw-Curtis quadrature rule, which is based on an expansion of the integrand in terms of Chebyshev polynomials, or evaluate the integrals numerically. The Clenshaw-Curtis quadrature rule is described in detail by Zhang \& Oosterlee (2013).

\section{Appendix B: Additional Calibration Experiment}

For the dataset described in Sec. 5, we perform an additional calibration experiment. We calibrate the time-dependent FX-SABR model to expiries 1, 2, 3 and 4 years. Results are provided in Fig. B.1. Except for the first expiry, the calibration of the time-dependent model is accurate. Also for the first expiry, the improvement of the time-dependent model compared to the constant-parameter model is significant.
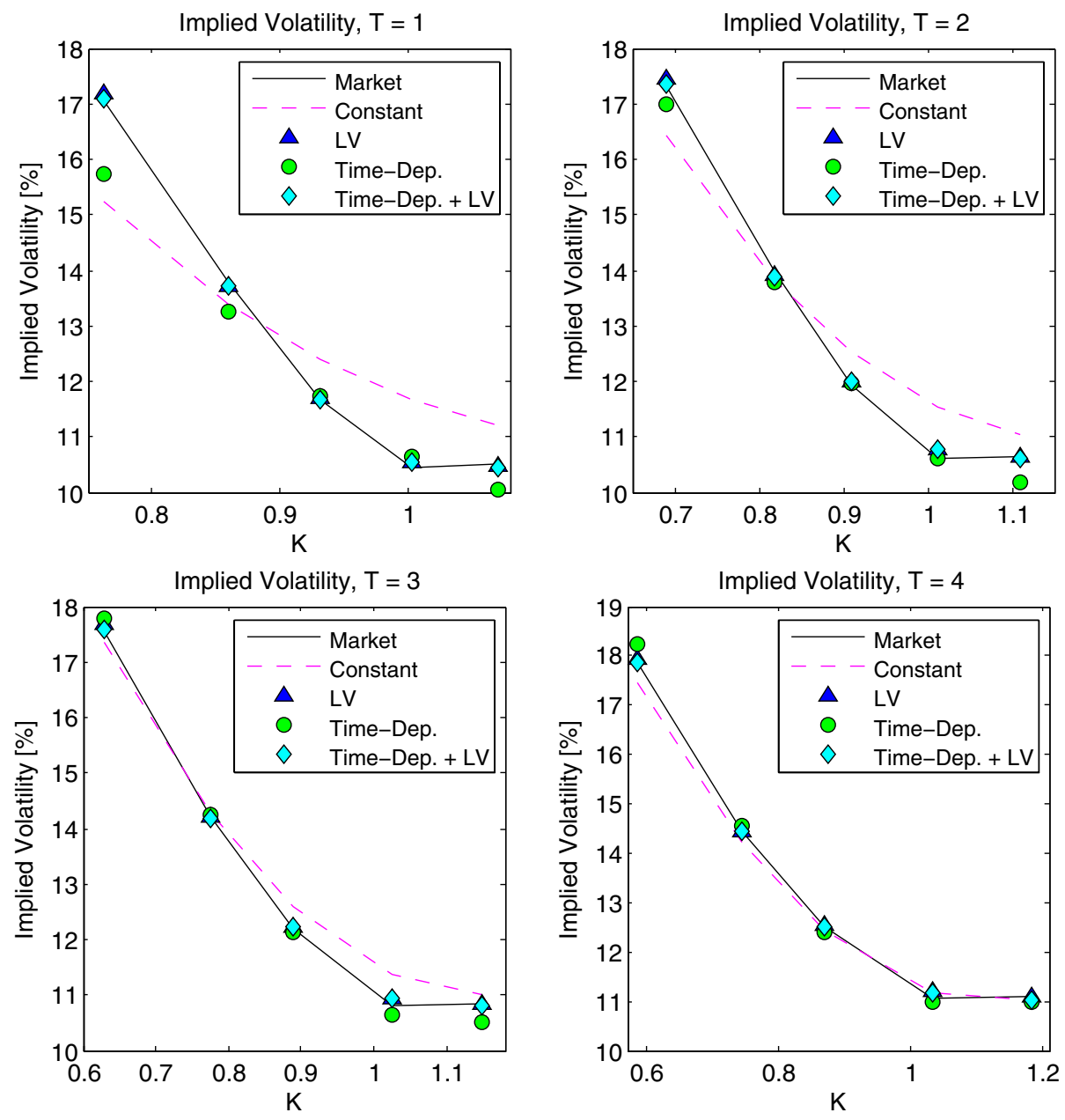

Fig. B.1. Black-Scholes implied volatility smiles for 1, 2, 3 and 4 years. 


\section{References}

A. Antonov, M. Konikov \& M. Spector (2013) SABR spreads its wings, Risk 26 (8), 58-63.

G. Baker, R. Beneder \& A. Zilber (2004) FX barriers with smile dynamics, Available at SSRN 964627.

B. Chen, L. A. Grzelak \& C. W. Oosterlee (2012) Calibration and Monte Carlo pricing of the SABR-Hull-White model for long-maturity equity derivatives, Journal of Computational Finance 15 (4), 79-113.

B. Chen, C. W. Oosterlee \& H. Van der Weide. A low-bias simulation scheme for the SABR stochastic volatility model, International Journal of Theoretical and Applied Finance 15 (2), 1-37.

J. Cox (1975) Notes on option pricing I: Constant elasticity of diffusions, Unpublished draft.

M. Curran (1994) Valuing Asian and portfolio options by conditioning on the geometric mean price, Management Science 40 (12), 1705-1711.

G. Deelstra, J. Liinev \& M. Vanmaele (2004) Pricing of arithmetic basket options by conditioning, Insurance: Mathematics and Economics 34 (1), 55-77.

E. Derman, D. Ergener \& I. Kani (1995) Static options replication, The Journal of Derivatives 2 (4), 78-95.

E. Derman \& I. Kani (1998) Stochastic implied trees: Arbitrage pricing with stochastic term and strike structure of volatility, International Journal of Theoretical and Applied Finance 1 (1), 61-110.

B. Dupire (1994) Pricing with a smile, Risk 7 (1), 18-20.

B. Engelmann, F. Koster \& D. Oeltz (2011) Calibration of the heston stochastic local volatility model: A finite volume scheme, Available at SSRN 1823769.

F. Fang \& C. W. Oosterlee (2008) A novel pricing method for European options based on Fourier-Cosine series expansions, SIAM Journal on Scientific Computing 31 (2), 826-848.

J. Fernández, A. Ferreiro, J. García, A. Leitao, J. López-Salas \& C. Vázquez (2013) Static and dynamic SABR stochastic volatility models: Calibration and option pricing using GPUs, Mathematics and Computers in Simulation 94, 55-75.

R. Frey \& D. Sommer (1996) A systematic approach to pricing and hedging international derivatives with interest rate risk: Analysis of international derivatives under stochastic interest rates, Applied Mathematical Finance 3 (4), 295-317.

J. Gatheral (2011) The Volatility Surface: A Practitioner's Guide. Vol. 357, John Wiley \& Sons, Ltd.

H. Geman, N. El Karoui \& J.-C. Rochet (1995) Changes of numéraire, changes of probability measure and option pricing, Journal of Applied Probability 32 (2), 443-458.

P. Glasserman \& Q. Wu (2011) Forward and future implied volatility, International Journal of Theoretical and Applied Finance 14 (3), 407-432.

L. A. Grzelak \& C. W. Oosterlee (2012) On cross-currency models with stochastic volatility and correlated interest rates, Applied Mathematical Finance 19 (1), 1-35.

I. Gyöngy (1986) Mimicking the one-dimensional marginal distributions of processes having an Itô differential, Probability Theory and Related Fields 71 (4), 501-516.

P. S. Hagan, D. Kumar, A. S. Lesniewski \& D. E. Woodward (2002) Managing smile risk, Wilmott Magazine 84-108.

J. Hull \& A. White (1993) One-factor interest-rate models and the valuation of interestrate derivative securities, Journal of Financial and Quantitative Analysis 28 (02), $235-254$. 
O. Islah (2009) Solving SABR in exact form and unifying it with LIBOR market model, Available at SSRN 1489428.

S. Johnson \& H. Lee (2003) Capturing the smile, Risk 16 (3), 89-95.

I. Karatzas and S. Shreve (1991) Brownian Motion and Stochastic Calculus. Vol. 113, Springer Science \& Business Media.

A. Kawai \& P. Jäckel (2007) An asymptotic FX option formula in the cross currency libor market model, Willmot Magazine March, 74-84.

K. Larsson (2010) Dynamic extensions and probabilistic expansions of the SABR model. Available at SSRN 1536471.

R. Lord (2006) Partially exact and bounded approximations for arithmetic Asian options, Journal of Computational Finance 10 (2), 1.

M. Musiela \& M. Rutkowski (2006) Martingale Methods in Financial Modelling, Vol. 36. Springer Science \& Business Media.

J. Oblój (2008) Fine-tune your smile: Correction to Hagan et al., Wilmott Magazine 35, 102-104.

Y. Osajima (2007) The asymptotic expansion formula of implied volatility for dynamic SABR model and FX hybrid model, Available at SSRN 965265.

L. Paulot (2009) Asymptotic implied volatility at the second order with application to the SABR model, Available at SSRN 1413649.

V. Piterbarg (2005) Time to smile, Risk 18 (5), 71-75.

V. Piterbarg (2006) Cross-currency exotics: Smiling hybrids, Risk 19 (5), 66-71.

R. Rebonato (2005) Volatility and Correlation: The Perfect Hedger and the Fox. John Wiley \& Sons.

R. Rebonato, K. McKay \& R. White (2011) The SABR/LIBOR Market Model: Pricing, Calibration and Hedging for Complex Interest-Rate Derivatives. Wiley.com.

Y. Ren, D. Madan \& M. Q. Qian (2007) Calibrating and pricing with embedded local volatility models, Risk 20 (9), 138-143.

S. E. Shreve (2004) Stochastic Calculus for Finance II: Continuous-Time Models. Vol. 11, Springer Science \& Business Media.

J. Sippel \& S. Ohkoshi (2002) All power to PRDC notes, Risk 15 (11), 1-3.

A. Takahashi, K. Takehara \& M. Toda (2007) Computation in an Asymptotic Expansion Method, John and Wiley \& Sons, Ltd.

H. Tanaka (1963) Note on continuous additive functionals of the 1-dimensional Brownian path, Z. Wahrscheinlichkeitstheorie 1, 251-257.

G. Tataru \& T. Fisher (2010) Stochastic local volatility, Quantitative Development Group, Bloomberg Version 1.

A. W. van der Stoep, L. A. Grzelak \& C. W. Oosterlee (2014) The Heston stochasticlocal volatility model: Efficient Monte Carlo simulation, International Journal of Theoretical and Applied Finance 11 (7), 1450045.

A. Van Haastrecht \& A. Pelsser (2011) Generic pricing of FX, inflation and stock options under stochastic interest rates and stochastic volatility, Quantitative Finance 11 (5), 665-691.

T. Weithers (2011) Foreign Exchange: A Practical Guide to the FX Markets, Vol. 309. John Wiley \& Sons.

Q. Wu (2012) Series expansion of the SABR joint density, Mathematical Finance 22 (2), 310-345.

B. Zhang \& C. W. Oosterlee (2013) Efficient pricing of European-style Asian options under exponential Lévy processes based on Fourier Cosine expansions, SIAM Journal on Financial Mathematics 4 (1), 399-426. 